\title{
MODELOS PROBABILÍSTICOS MULTINOMIALES PARA EL ESTUDIO DEL CECEO, SESEO Y DISTINCIÓN DE /S/ Y $/ \theta /$. DATOS DE LA CIUDAD DE MÁLAGA
}

\author{
J. A. VILLENA PONSODA - J. M. SÁNCHEZ SÁEZ - A. ÁVILA MUÑOZ \\ (Universidad de Málaga)
}

\begin{abstract}
RESUMEN
The aim of this paper is to propose a multinomial probabilistic model able to account for the variation of $/ \theta^{s /}$ in Southern Spanish varieties. The current analyses carried up till now are based on frequency counts of mergers between $/ \mathrm{s} /$ and $/ \theta /$, which are labeled 'ceceo' and 'seseo', as two social and regional norms of pronunciation. Standard Spanish distinction between $/ \mathrm{s} /$ and $/ \theta /$ is therefore to be deduced from the total body of cases, counting every [s] corresponding to an etymological or canonical $s$ as an occurrence of $/ \mathrm{s} /$, including the tokens of those individuals whose pronunciation pattern of $/ \theta^{\mathrm{S}} /$ is overtly 'seseo' (exactly the same for the contrary case of 'ceceo').

Since both types ( $s>\theta$ and $\theta>s$ ) currently coexist in Southem speech communities as in Málaga city, it is not obvious to decide about the high proportion of cases where $/ \mathrm{s} /$ and $/ \theta /$ are pronounced in a canonical way. Therefore we need a procedure to decide the rate of cases of canonical pronunciation due to chance.

Two related multinomial models have been developed, implemented and tested on a sociological body of data from a social network of 31 individuals from Málaga city, extracted from a sociolinguistic investigation of vernacular varieties in Málaga (M.U.V.-Project). The first model calculates the single probability of seseo $(\mathrm{s}>\mathrm{s}$ and $\theta>\mathrm{s})$, ceceo $(\theta>\theta$ and $\mathrm{s}>\theta)$ and distinction $(\mathrm{s}>$ $s$ and $\theta>\theta$ ) for every individual studied. The second model calculates the single probability of distinction ( $s>s$ and $\theta>\theta$ ) vs. the single probability of reduction or 'confusión' ( $s>\theta$ and $\theta>s$ ).

To measure the degree of fit of both models, a maximum likelihood estimator has been developed with consistent and hopeful results. Comparison between
\end{abstract}

Este trabajo se ha realizado en el marco del Proyecto de Investigación del Sistema de Variedades Vernáculas Malagueñas (Proyecto V.U.M.), financiado por la DGICYT PB91-0417. 
relative frequency and probabilistic results demonstrates, finally, the more realistic approach to this problem on the basis presented here

\section{Introducción}

En este trabajo se proponen dos modelos probabilísticos basados en la distribución multinomial, uno para el estudio de los fenómenos de seseo, ceceo y distinción de $|\mathrm{s}| \mathrm{y}|\theta|$, y el otro para el estudio de la confusión-distinción de $|\mathrm{s}|$ y $|\theta|$. El objetivo del desarrollo de estos modelos es estimar el comportamiento lingǘstico de los sujetos investigados, a partir de los datos obtenidos en una muestra de conversación dirigida obtenida en su contexto más usual. Se mostrará una investigación referida a un conjunto de individuos del barrio de Capuchinos de Málaga. El desarrollo de estos modelos, así como los datos utilizados para la aplicación, se localizan en el contexto del Proyecto de Investigación del Sistema de Variedades Vernáculas Malagueñas (Proyecto V.U.M.), que se desarrolla en el Departamento de Filología Española I de la Universidad de Málaga.

Se puede encontrar un amplio estudio sociolingǘstico de la red social de la que se ha extraído los datos, así como la explicación de los resultados obtenidos a partir de los modelos probabilísticos que se van a desarrollar en este trabajo, en los de Villena y Requena (1994) y Ávila (1994).

\section{La situación sociolingüística de $s / \theta$ en el español meridional.}

2.1. La diferenciación sociolingüística manifestada a través de rasgos fonéticos del subsistema de consonantes palatales y dentales $\left(/ \theta^{\mathrm{s}} /, / \hat{\mathrm{s}} /, / \mathrm{y} /\right)$ en el español hablado en la ciudad de Málaga es un asunto observado desde antiguo (Alvar 1973, 12-13; Navarro Tomás et alii 1933, 53-54)1, en correspondencia con observaciones similares para el resto del dominio meridional español: la variable $/ \theta \mathrm{s} /$, procedente de las cuatro sibilantes del español medieval $/ \dot{\mathrm{s}} /,|\dot{\mathrm{z}}|$, $/ \$ /, / \hat{J} /$, se realiza mediante variados alófonos mates (moderno ceceo, como en [pá

El ALEA VI m. 1705, lám. 1580, apunta para Málaga capital (Ma 406) las siguientes nommas de pronunciación: seseo en las mujeres y los hablantes cultos del casco urbano; ceceo en los hablantes de las zonas periurbanas rurales; seseo-ceceo y ceceo-seseo en los hombres jóvenes y de cultura baja. La situación varía en las distintas capitales, según las zonas geograficolingüísticas (seseo, ceceo o distinción).

2 Los matices de realización de ambos géneros de pronunciación son muy amplios ([s]---[h]) $([\theta]--[h])$, como se puede observar en el ALEA, VI. Galeote (1988, 35-70) ha hecho una descripción exhaustiva de las variantes de $/ \mathbf{s} /\left(\underline{s}, \underline{s}, \underline{s}^{z}\right.$, etc. $)$ y de $/ \theta /\left(\theta, \theta^{s}\right.$, etc. $)$ y las ha cruzado con el sexo y la ocupación de sus setenta y cinco informantes del treviño de Iznáar, Villanueva de Tapia y Venta de Santa Bárbara, en el encuentro de las provincias de Málaga, Granada y Córdoba 
asociándose, desde siempre, los primeros con la ausencia o deficiencia de instrucción formal y el origen rural, y los segundos con la cultura urbana y la educación formal (Alvar 1974, 18-19; Navarro Tomás 1933, 23-60). Se trata, en estos casos, de normas, ejemplos o modelos ideales de pronunciación, pero no naturalmente de pautas homogéneas; de hecho, la mezcla y alternancia caótica de realizaciones (trueque anárquico: seseo-ceceo / ceceo-seseo; ceseo / seceo) 3 puede llegar a constituir una pauta alternativa (aunque es muy dudoso que pudiera considerarse como una norma, en sentido estricto).

El fenómeno de confusión, desfonologización (Carbonero 1982), neutralización (ALEA), igualación fonológica (Llorente 1962) o reducción (Sawoff 1980) entre /s/y / $/$ / es un asunto profusamente investigado, y sin duda bien conocido en todas sus etapas y aspectos, desde el punto de vista diacrónico y geolingüístico (Alvar, Alonso, Ariza, Catalán, Cock Hincapié, Frago, Guitarte, Lapesa, Menéndez Pidal, Mondéjar, etc. $)^{4}$. En la Andalucía confun-

En el presente trabajo sólo se consideran dos géneros de pronunciación: [ $\theta$ ], variante continua mate (sea interdental o dentointerdental, incluyendo teóricamente variantes levemente aspiradas o sonorizadas) y [s], variante continua estridente (sea coronoalveolar o predorsodental). $\mathrm{La}\left[\theta^{\mathrm{s}}\right]$ o $\left[\mathrm{s}^{\mathrm{\theta}}\right]$, que incluye las variantes intermedias y recoge los abundantes casos dudosos al ódo de los transcriptores, no se ha tenido en cuenta a efectos de los cálculos, habida cuenta de su menor frecuencia ( $1 \%$ de los casos, frente a $62 \%$ de [s] y $37 \%$ de [ $\theta$ ], para un total de 4777 ocurrencias de $/ \theta^{\mathrm{s}} \%$. Véase Avila 1994, caps. 3 y $4,40-72$.

3 "Creo oportuno indicar -escribe Llorente (1962, 230-231)-que actualmente, en toda el área de ceceo y en las zonas limítrofes que distinguen, pero donde sólo se conoce la s predorsal (ş), incluyendo las zonas urbanas consideradas seseantes enclavadas en el dominio rústico del ceceo, hay una gran anarquía fonética, pronunciándose indistintamente ş y $\theta$ (relajada), pues se ha operado una igualación fonológica representada por el archifonema $/ \theta^{s} /$ que tiene las tres siguientes realizaciones: $s, \theta, \theta^{5}(\ldots)$. La antigua riqueza fonológica se ha reducido a un solo fonema, mientras que, por el contrario, la pretendida uniformización fonética (ceceo o seseo) en muchas zonas no es tal, porque lo normal es la triple realización indicada". Para Sawoff (1980, 239-241), "... the heterogeneous pronunciation of Sevillan will, I think, clearly emerge from the following pages (...) The possibilities ... were simple in terms of phonology but complicated phonetically: either the stabilization around some old (or new) version of the Andalusian phonemic reduction of standard Spanish $/ \mathrm{s} /$ and $/ \theta /$ to one phoneme, or a phonemic retrograde development to "distinción", i.e., Standard-Spanish-Distinction between $/ \mathrm{s} /$ and $/ \theta /$ (...) The first two systems, seseo and ceceo, are well known... [junto a ellos, hay que distinguir un tercer sistema fonológicamente confundidor de $/ \mathrm{s} /$ y $/ \theta /$, que representa] ... "the sibilant system of all those Andalusians who do not use ceceo, seseo or SSD with any consistency. I would suggest the name "seceo" [seӨéo] for this hitherto rather neglected possibility ... It is, like seseo and ceceo, the phonemic reduction of standard Spanish $/ \mathrm{s} /$ and $/ \theta /$ to one phoneme, with the two ends of the articulatory continuum $[s]$ and $[\theta]$, being the two allophones in free variation ...". Vid. asimismo $\mathrm{P}$. Carbonero, "Polimorfismo y funcionalidad en el uso lingüístico de hablantes andaluces", in Sociolingiǘstica andahza 1, Servicio de Publicaciones, Universidad de Sevilla. ${ }^{2} 1985$ [1982]: 4756. pp. 49-53; Sawoff 1980, 247-253.

${ }^{4}$ La bibliografia sobre este problema es muy amplia. Véanse, por ej., M. Alvar, "A vucltas con el scseo y el ceceo", Estudios dedicados a D. Gazdanu, Romanica 5, 1972, Inst. de Filología, La Plata, 1974, 41-57; J. Mondéjar, "La más antigua caracterización fonética de las hablas andaluzas", in Dialectología andaluza. Estudios, Don Qujjote, Granada, 1991, 167-211; "Discuisiciones historicocríticas y metodológicas sobre la interpretación de los datos en el estudio del "sesco", in Dialectologia andaluza, 320-335, J.A Frago, Historia de las hablas andaluzas, 
didora de $/ \mathrm{s} /=/ \theta /$, en la que se incluye Málaga, se ha de partir de un fonema único; éste es, históricamente, $/ \mathrm{s} /$, consonante continua dorsodental sorda, procedente de la desfonologización de la pareja /z/ : / $/$ / continuas, dorsodentales sorda y sonora), y ésta, a su vez, de la reducción, respectivamente, de las oposiciones $/ z /$ : / $/$ / (continuas sonoras, dorsodental y apicoalveolar) y $/ s /: / \mathrm{s} /$ (continuas sordas, dorsodental y apicoalveolar), tras la previa pérdida de la distinción más estable basada en el rasgo de interrupción/continuidad $(/ \hat{s} /: / \dot{s} / \mathrm{y} / \hat{z} /: / \dot{z} /)^{5}$. La $/$ ş/, en la que confluyen las cuatro clases de palabras del español medieval con $/ \mathrm{s} /, \mid \dot{z} /, / \hat{s} /$ y $/ \hat{z} /$ (osso, oso, foçes, fozes), tiene un amplio campo de dispersión para la realización alofónica, que va desde las variantes estridentes $(s, \underline{s})$ a las mates $(\theta, \theta)$ : [ó $\theta$ o, óso, óşo]. Por el contrario, la solución del español ejemplar y de las variedades dialectales centroseptentrionales (incluidas las áreas septentrionales y orientales andaluzas), frente al problema descrito a partir de la fricatización de $\mid \widehat{s} />/$ ş $/$ y de $|\hat{z} />| z ̧ /$, consiste, como se sabe, en el refuerzo de la distinción secundaria basada en el punto de articulación de las parejas opuestas (orden palatal / dental; i.e, apicoalveolar y dorsodental), para llegar, por último a la oposición de dos unidades fonológicas: $/ \mathrm{s} /: \mid \theta /$.

Desde el punto de vista sincrónico, se han considerado con detenimiento las diferencias $\mathrm{y}$ distancias estructurales entre las soluciones meridionales $\mathrm{y}$ americanas $(/ \mathrm{s} /=/ \theta /)$ y las septentrionales y ejemplares peninsulares $(/ \mathrm{s} /: / \theta /)$, poniéndose de manifiesto, además, el carácter relacionado de, por un lado, la reducción meridional, canaria y americana $y$, por otro, los cambios en marcha en el orden palatal $(/ \hat{S} /: / y /)^{6}$. Lo que interesa destacar aquí es que, en la zona meridional - sea cual sea la norma de pronunciación adoptada, incluida la de distinción de $/ \mathrm{s} / \mathrm{y} / \theta /-$, el orden palatal queda reducido a una sola pareja de

Arco Libros, Madrid, 1993; "Variación dialectal y sociocultural en la documentación indiana del siglo XVI", RFE 72 (1992), 399-427; M. Ariza, "De la llamada revolución fonológica del Siglo de Or", in Sobre fonética histónica del español, Arco/Libros, Madrid, 1994, 223-257. Sobre la geografia lingüistica del fenómeno, cf. Navarro Tomás 1933; M. Alvar, "Modalidades fonéticas cordobesas", in Estudios de Geografia Linguística, Paraninfo, Madrid, 1991, 232-245; J. McMenamin, "Geografia dialectal y sociolingüística: un ejemplo andaluz", NRFH 27/2 (1978), 276-296; Galeote 1987, 35-70 y 149-166; J.B. Dalbor, "Observations on present-day seseo and ceceo in Southern Spain", Hispania 63 (1980), 5-19.

5 Mondéjar, "La más antigua caracterización", 171-189. Cf. Á. López, "Los reajustes fonológicos del español a la luz de una teoria del cambio", Estudios Filológicos en homenaje a $E$. de Bustos Tovar, II, Salamanca, 1993, 519-530.

- CF. R. Trujillo, "Cuestiones de fonología diatópica: dentales y palatales en el ALEA y en el ALEICan", in Philologica Hispaniensia. Homenaje a M. Alvar, I, Dialectología, Madrid, 1983 , 609-621; J.A Moya, "Problemas fonológicos del orden palatal", Studia Litteraria atque Linguistica N. Marín, J. Fennández Sevilla et P. González Oblata, Servicio de Publicaciones, Universidad de Granada, 1988, 151-171, donde se analizan en profundidad los problemas estructurales y se cita y se comenta la bibliografia más importante. 
fonemas opuestos ( $\hat{\mathrm{s}} / \mathrm{y} / \mathrm{y} /)$, pasando la continua sorda estridente (s etimológica confundida, como se señaló arriba, con la s) al orden dental (donde, dadas las realizaciones, parece apropiado notar dicha unidad fonémica como $\left./ \theta^{s} /\right)$ :
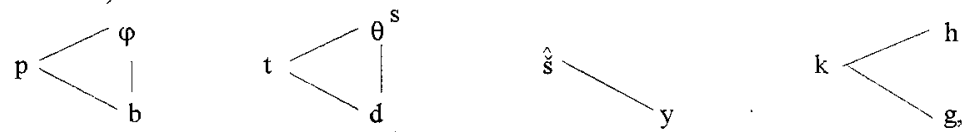

frente a la solución centroseptentrional y ejemplar peninsular, en la que la /s/ pertenece al orden palatal, como es notorio ${ }^{7}$ :
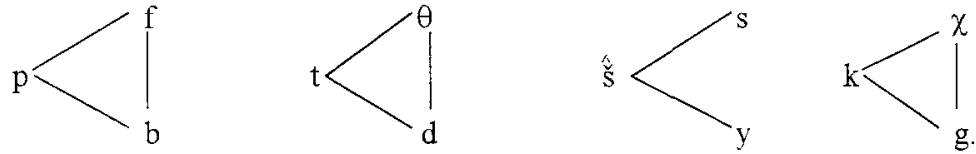

Junto al modelo meridional confundidor, encontramos asimismo soluciones conservadoras de la distinción entre $/ \mathrm{s} / \mathrm{L} / \theta /$; se trata, por un lado, de localidades septentrionales andaluzas, cuya adscripción a las variedades extremeñas, manchegas o murcianas es indudable, y en las que, por lo tanto, la $/ \mathrm{s} /$ presenta realizaciones palatales (apicoalveolares) ${ }^{8}$; por otro lado, puede manejarse un modelo de distinción con géneros de pronunciación no palatales para la /s/ (dorsodentales; coronoalveolares, etc.). De este modo, la $/ \mathrm{s} /$ (continua, dental, estridente) se diferencia de la $/ \theta /$ (continua, dental, mate) de modo muy precario (y con el mantenimiento paralelo de la oposición entre $/ \hat{\mathrm{s}} / \mathrm{y}$ /y/ basada, a la vez, en los rasgos de sonoridad o tensión y de interrupción, como en el español ejemplar, frente a su diversificación meridional: $/ \check{s} /: / 2 \varnothing / \mathrm{y}$

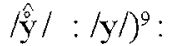
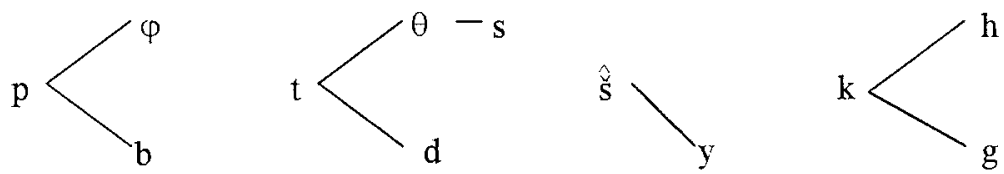

Lo que, desde luego, conviene notar es la falta de consistencia (seguramente parangonable con lo sucedido tiempo atrás en América) ${ }^{10}$, no sólo ya en la realización normal de la unidad $/ \theta^{\mathrm{S} /}$ (esto es, los cambios -o, valdría mejor decir, correspondencias diasistemáticas- de $\mathrm{s}>\theta$ y $\theta>\mathrm{s}$, en

\footnotetext{
7 Cf. E. Alarcos, Fonología española, Gredos, Madrid, 1968, 169-179.

$8 \quad$ Cf., por ej., Alvar, "Modalidades cordobesas", supra cit., 237-241.

- Vid. Moya, "Problemas fonológicos del orden palatal", supra cit, 155-160 et passim.

10 Véase G. Guitarte, "Seseo y distinción s-z en América durante el siglo XIX", in Siete enscryos sobre el español de América, UNAM, México, 1983, 107-125; cf. Caravedo 1992, 640642.
} 
aparente variación libre, frente a las pautas regulares de seseo y ceceo), sino, fundamentalmente, en el paso o alternancia inter e intraindividual de los modelos de reducción $(/ \mathrm{s} /=/ \theta /)$ a los de distinción $(/ \mathrm{s} /: / \theta /)$ y viceversa, en áreas geográficas como la de la ciudad de Málaga, en las que, como se ha dicho, los susodichos fonemas sibilantes medievales se redujeron tempranamente a uno solo ${ }^{11}$. Así, por un lado: $/ \theta^{\mathrm{S}} /$, como en $/$ pé $\theta^{\mathrm{S}} \mathrm{o} /, \theta^{\mathrm{S}}$ apáto/, $/ \operatorname{ta} \theta^{\mathrm{s}} \mathrm{a} /$ (peso, zapato, taza, tasa), con realización dorsodental estridente [péşo, şapáto, táşa] o dentointerdental mate [péச,o, /s/, como en /péso/, / $\theta$ apáto/, /tása/, /tá $\theta$ a/ (peso, zapato, taza, tasa), con las mismas realizaciones (continua sorda dorsodental estridente o dentointerdental sorda mate). La diferencia, sin embargo, estriba en que, en el modelo de distinción sui generis que se da en las áreas meridionales (en el que aparecen sistemáticamente variantes dentales de $/ \mathrm{s} /)^{12}$, las realizaciones están -como en el español ejemplar peninsular- fijadas a los contextos canónicos (etimología), como en [ $\theta$ apáto], [péso], y/o distinguen de por sí pares mínimos, como en [tása], [tá $\theta$ a], mientras que en el modelo de reducción o confusión dicha fijación no existe o es sólo parcial ${ }^{13}$ :

11 CE M. Alvar Ezquerra, Conquista, emigración, repoblación y habla, Discurso de recepción en la Real Academia de Bellas Artes de San Telmo, Málaga, 1994.

12 De hecho, la oposición funciona y es reconocible sistemáticamente cuando se realiza con variantes apicoalveolares o coronales planas (A. Quilis, Fonética acústica de la lengua española, Gredos, Madrid, 1981, 229-239 y 255), mientras que hay datos acústicos patentes de que el margen de seguridad entre $/ \theta /$ interdental o dentointerdental y $/ \mathbf{s}$ dorsodental es muy precario (dada la tendencia de ésta última a perder la estridencia que caracteriza a la $/ \mathbf{s} /$ : "A medida que el lugar de articulación va avanzando y se sitúa en la proximidad dental, la estridencia va disminuyendo, dejando paso a la cualidad de mate, que se hace patente en el espectro de la [s] predorsodentoalveolar [...] La característica mate lleva consigo una distribución más regular de las regiones de frecuencias, distribución que origina unos espectros semejantes a los de [ $\theta]^{\prime \prime}$ (ibid., 236).

13 Cf. Caravedo (1992, 644-646 y 650-654), donde se diferencia, a este propósito, entre la distinción, la indistinción y la indistinción parcial. Las zonas funcionales (o espacios permisibles de realización fónica) de las unidades (aquí $/ \mathrm{s} / \mathrm{y} / \theta /$ ) admiten un campo, radio o espacio de variabilidad, siempre que ésta no sobrepase los límites de los espacios limítrofes. Los fonemas se distinguen de modo constante en la medida en que se fijan a determinados contextos léxicos canónicos; en mucha menor medida en cuanto que pueden diferenciar significados lingüísticos (pares mínimos). Los procesos de indistinción consisten en la invasión del campo funcional del fonema opuesto, mediante el desajuste de la fjación contextual aludida (s y z; y/li $\mathrm{m} / \mathrm{\text {,etc.) }}$. Precisamente, el problema que presenta el estudio cuantitativo de la variación de $1 \theta^{\mathrm{s}} /$ es el del correcto tratamiento de las pautas de indistinción parcial; esto es, "una fase de parcialidad o variabilidad distintiva en la que se mezclan o superponen los patrones y las zonas funcionales" (p. 652). Vid asimismo, R. Caravedo, Sociolingüística del español de Lima, Pontificia Univ. Católica del Perú, Lima, 1990, 41-69; "Los espacios de variabilidad en fonología", Voz y Letra $2 / 1$ (1991), 17-38. En cuanto al interesante trabajo (1992) sobre la conservación de restos de la distinción $s / \theta$ en los Andes peruanos (sobre el que se volverá después), se dan algunas premisas y circunstancias específicas que singularizan el problema en relación con el mismo en Andalucía: $\left.1^{\circ}\right)$ la oposición constatada en Cajamarca y Cuzco se establece entre variantes apicoalveolares de is e interdentales de $/ \theta /$, frente a la situación andaluza, en la que se manejan por lo común (y 
NORMAS

\begin{tabular}{|l|c|c|}
\multicolumn{1}{c}{} & $\begin{array}{c}\text { REDUCCIÓN } \\
/ \theta /=/ \mathrm{s} /\end{array}$ & $\begin{array}{c}\text { DISTINCIÓN } \\
/ \theta /: / \mathrm{s} /\end{array}$ \\
\hline FIJACIÓN & $/$ pé $\theta^{\mathrm{s}} \mathrm{o} /$ & $/$ péso/ \\
CONTEXTUAL & $/ \theta$ apáto/ \\
& $/ \theta^{\mathrm{s}}$ apáto/ & \\
\hline PARES MÍNIMOS & $/$ tá $\theta^{\mathrm{s}} \mathrm{a}$ & $/$ tása/:/tá $\mathrm{a} /$ \\
\hline
\end{tabular}

seseo: dental estridente ceceo: dental mate distinción: estridente/mate

$\begin{array}{ccc}{[\mathrm{s}]} & {[\theta]} & / \theta /: / \mathrm{s} / \\ {[\text { péso] }} & {[\text { pé } \theta \text { o] }} & {[\text { péso] }} \\ {[\text { sapáto] }} & {[\theta \text { apáto] }} & {[\theta \text { apáto] }} \\ \ldots \ldots . . & \ldots \ldots \ldots & \ldots \ldots \ldots \\ {[\text { tása }]} & {[\text { tá } \theta \mathbf{a}]} & {[\text { tása] } /[\text { tá } \theta \mathbf{a}]} \\ {[\text { tása }]} & {[\text { tá } \theta \mathbf{a}]} & \end{array}$

2.2. La aludida alternancia entre los modelos comentados (o indistinción parcial entre $/ \theta / \mathrm{y} / \mathrm{s} /$ ) posiblemente deba atribuirse - frente a lo que ocurre en otras zonas- a la «vigencia del patrón distinguidor como prestigioso en la misma nación», como señala R. Caravedo (1992, 641). A dicha razón responderían, de hecho, las frecuentes observaciones de los dialectólogos y

salvo en las zonas comentadas arriba) alófonos dorsodentales de $/ \mathrm{s} /$ y dentointerdentales de $/ \theta /$; $2^{\circ}$ ) la indistinción completa constatada por Caravedo para el español de la costa peruana (esto es,

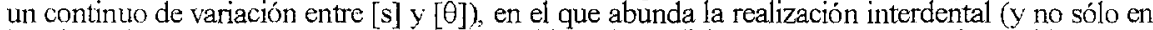
las clases bajas) no merece, según ella, ser objeto de análisis porque "no se puede establecer una separación clara, tajante y recurrente entre los dos tipos articulatorios como ocurre en la modalidad andina ..." (pág. 643); no obstante, en esa distinción se ejercitan muchos de los hablantes cultos de Andalucía, sin por ello cambiar la articulación de la /s/ ("Sin embargo -según Trujillo, "Cuestiones de fonología diatópica", supra cit., 612-, no parece que fuera necesaria la confusión de ambos fonemas por tal motivo [el adelantamiento de la s tras los cambios entre las fricativas sibilantes medievales], ya que cabe una solución estridente / mate $([\mathrm{s}]$ - $[\theta])$, no hipotética sino real [...] El contraste estridente mate deja de ser redundante y asume la función distintiva. Es así como existe un sistema -con gran probabilidad de transición- donde $s / \theta$ se oponen sobre las características fonológicas comunes de sordas fricativas dentales"); $3^{\circ}$ ) se consideran únicamente los casos de fijación contextual de $z>\theta$ (frente a $z>s$ ), pero no los de $s>s$ (frente a $s>\theta$ ), puesto que se parte de la base común americana de la generalización de alófonos estridentes; tal es un requisito indispensable para hablar de indistinción total (seseo, con fijaciones contextuales canónicas cercanas a cero); sin embargo, y dada la variación costeña (por lo que parece, similar a la andaluza) podría ser de interés comprobar en qué medida no se da dichà fijación. 
sociolingüistas sobre el particular, en el sentido de que la proporción de distinción captada en sus informantes confundidores o reductores (mayoritariamente ceceantes o seseantes) constituye el resultado de una elección fuertemente condicionada por la formalidad de la situación (Alvar 1974, 19) ${ }^{14}$ y por la educación del hablante o su edad (Ávila 1994; Carbonero et alii 1992, 23-25; Moya y García Wiedemann, en prensa). En cuanto a los posibles condicionamientos internos lingüísticos poco se sabe, de momento, aunque no es descartable su influencia en el desarrollo de los cambios parciales de $\mathrm{s}>\theta \mathrm{y}$ de $\theta>\mathrm{s}^{15}$.

Existen pocos estudios cuantitativos sincrónicos sobre el problema de la realización de $/ \theta \mathrm{s}$, así como sobre el más general de la alternancia inter e intrapersonal de los modelos sociofonológicos de las sibilantes en el español meridional. Tras el conocido trabajo de Navarro Tomás sobre la frontera del andaluz (1933), Alvar $(1973,1974)$ y Llorente (1962) se ocuparon del problema, al establecer áreas fonéticas interiores y al proponer interpretaciones variadas de los datos del ALEA. Con posterioridad, se han realizado algunas aproximaciones de interés, particularmente centradas en el ámbito urbano y, en general, basadas metodológicamente —más o menos de cerca, más o menos explícitamente- en los presupuestos del PILEI para el proyecto de estudio de la norma culta del español general ${ }^{16}$. Se dispone, en consecuencia, de un mapa sociolingǘstico fragmentario, y con diferentes grados de profundidad, sobre la citada parcela del sistema fonológico del español de Andalucía, que aclara, sin embargo, algunos aspectos tenidos por oscuros anteriormente.

En Sevilla, P. Carbonero $(1982,1985)$ se ocupó tempranamente del problema, en el marco de la determinación empiricoestadística de la norma culta a partir de un conjunto de rasgos fónicos (aspiración y pérdida de $-\mathrm{s}$ en final de sílaba y sus consecuencias en el entorno fonético; aspiración de otras consonantes; reducción de $/ \mathrm{s} /=/ \theta /$ y realización seseante o ceceante; igualación de $/ \mathrm{r} /=/ 1 /$ pérdida de $/ \mathrm{r} / \mathrm{o} / \mathrm{l} /$ en final de palabra; variación entre $[\chi]$ y [h]), y los puntuó de acuerdo con su nivel o índice de aceptación (número relativo de hablantes que utilizan la realización típica), su homogeneidad (la uniformidad o falta de dispersión de las soluciones) y su seguridad (la regularidad o falta de vacilación en el uso individual). La indistinción de $s / \theta$

14 "Pues de nada sirve que un universitario, en una lectura -y ante un explorador que para él no dejaba de ser catedrático-, distinguiera de vez en cuando s y $z$, a la manera castellana, cuando lo que realizaba siempre era el seseo".

15 Este es, sin embargo, un problema diferente del que aquí concieme, y será objeto de atención en otro sitio. Véase, más adelante, párr. 2.3.

16 Vid, por ej., J.M. Lope Blanch. Estudios sobre el español hablado en las principales ciudades de América, UNAM, México, 1977, XV. 
muestra, según los datos sevillanos, una aceptación en el nivel culto de 0.70 (homogeneidad: 0.40 ; seguridad: 0.67 ), frente a, por ejemplo, la $-\mathrm{s}$ final de palabra $(0.90,0.81,0.90)$, o la neutralización de $r / 1(0.27,0.45,0.75)$; ello sugiere una amplia difusión y la presencia de variación inter e intraindividual de las normas de distinción y seseo, especialmente (1982, 146-150). En cuanto a la oposición entre seseo y ceceo $(1985,78-82)$, se informa sobre la amplia aceptación del seseo (nivel culto: 0.74 ; nivel medio: 0.86 ; nivel popular: 1; índice general: 0.87 ), frente a la baja consideración del ceceo (culto y medio: 0 ; popular: 0.19; índice general: 0.06).

Sawoff (1980) se basó en los datos del estudio del habla de Sevilla, así como en los obtenidos por él mismo en una investigación anónima en la ciudad, desarrollada a partir de un breve cuestionario previo (observación del cambio de $z$ etimológica $>\mathrm{s}$, frente al mantenimiento de $z$ etimológica $>\theta$ ). Recogió datos distinguiendo barrios, prestigio profesional (droguerías, tiendas de ultramarinos y bancos) y sexos. En los 82 sujetos estudiados en los dos primeros tipos de establecimientos, observó el cambio de $z>s$ en el 0.66 de las apariciones y el mantenimiento de $\mathrm{z}>\theta$ en el 0.34 restante, si bien resulta metodológicamente imposible decidir, en el segundo caso, si [ $\theta$ ] debe interpretarse como un ejemplo del modelo de pronunciación ceceante, distinguidor 0 , incluso, de las pautas intermedias (seceo, ceseo) ${ }^{17}$. En los bancos, por el contrario, los resultados fueron, respectivamente, 0.54 y 0.41 para $z>s$ y $z>\theta$ , a los que se añade el $0.04 \mathrm{de} z>\theta^{\mathrm{S}}$. La consideración de las diferencias geográficas urbanas (centro, periferia) y genéricas, muestra el aumento de la pronunciación fricativa mate $[\theta]$ de la $z$ etimológica en la periferia (en contraste con el centro urbano: 0.37 frente a 0.25 ) y, muy notablemente, de los hombres, frente a las mujeres $(0.36 / 0.00$ en la periferia y $0.50 / 0.22$ en el centro), para el caso de los sujetos de las droguerías y de los ultramarinos. En los bancos, los porcentajes se acercaron más (centro: $0.41 /$ periferia: 0.44 ), aunque es indudable que parte del aumento de $[\theta]$ deba atribuirse al seguimiento - irregular o dependiente del contexto- del modelo ejemplar de distinción, frente al grupo anterior en el que, según Sawoff (1980, 245-246), la interpretación va más en el sentido de ceceo de origen rural.

Con respecto a las diferencias de edad, Sawoff observó una notable tendencia de las generaciones intermedias (30-50 años), más consolidadas social y profesionalmente que las demás, al uso de la distinción española ejemplar ${ }^{18}(0.47$ y 0.33 de $z>s$, respectivamente, para los sujetos entre $30-40$

17 Sawoff 1980, 242-244. Véase, más adelante, párr. 2.5 y 3.

18 Spanish Standard Distinction (SSD). Conviene recordar que se trata de una importante suposición basada en las diferencias de prestigio profesional, en el origen (rural, urbano) y en la 
y 40-50 años), mientras que los más jóvenes (20-30 años) y los mayores (5060 años) alcanzaban porcentajes elevados de seseo ( 0.67 y 0.62$)$.

En Jerez, Carbonero y sus colaboradores (1992, 18 y 23-25) señalan índices de aceptación ${ }^{19}$ del 0.09 para el mantenimiento de la distinción de /s/y / $\theta /, 0.44$ para el seseo y 0.47 para el ceceo (indistinción: 0.91). Estos índices de distinción de $/ \mathrm{s} /: / \theta /$ se distribuyen de una manera homogénea y con valores muy bajos a lo largo de todas las categorías: $0.17,0.05,0.05$ para la $I^{\mathrm{a}}, \mathrm{II}^{\mathrm{a}}$ y $\mathrm{II}^{\mathrm{a}}$ generación, respectivamente; del mismo modo, para los diferentes niveles educativos: 0.12 en el nivel culto, 0.15 en el medio y 0.00 en el popular. Algo más notables son las diferencias genéricas: 0.14 para los hombres y 0.04 para las mujeres. El seseo $(\theta>\mathrm{s})$ se muestra como un rasgo relacionado con los grados superiores de instrucción formal $(0.76$ de $\theta>\mathrm{s}$ en el llamado nivel culto, frente al 0.12 de $\mathrm{s}>\theta$ o ceceo) y con las generaciones intermedias $(0.58$, frente a 0.37 ). Entre los jóvenes, desciende el seseo (0.33), mientras que el ceceo tiende a limitarse en beneficio de la distinción $(0.50$ y 0.17$)$. Los mayores, entre los cuales es el ceceo el rasgo más empleado (0.53), alcanzan cifras intermedias de seseo (0.42). Por último, las mujeres reafirman la conocida tendencia al seseo (0.49), frente a los hombres (0.40), aunque, debido a sus bajos índices de distinción, igualan a los hombres en el índice de ceceo (0.47 frente a 0.46$)$.

Los bajos índices generales de mantenimiento de $/ \mathrm{s} / \mathrm{y} / \theta /$ en Jerez, y su relativa subida entre los individuos de la $\mathrm{I}^{\mathrm{a}}$ generación, hacen pensar en que «la distinción $s$ y $z$ estuviera adquirida - aunque de manera no intensa- por los individuos más jóvenes de la población» (ibid., 24). Por otra parte, tampoco se encuentran indicios de que la oposición de $/ \mathrm{s} /: / \theta /$ tenga especial prestigio en Jerez; la confusión $/ \mathrm{s} /=/ \theta /$ obtiene un índice de aceptación general de 0.91 , mientras que el índice de aceptación de la distinción de ambos fonemas en el nivel culto sólo alcanza el 0.12 , incluso menor que el correspondiente a los hablantes del nivel medio, «el cual parece ser el más sensible a la hiperco-

situación geográfica urbana de los hablantes (centro y periferia). Según ésta, la [0] de, v. gr. [tranferenêia] ( $<$ z etimológica), podría interpretarse como SSD entre los sujetos de clase media y urbanos (i.e, proclives a realizar [s], por ej., en [mesa]), mientras que la misma solución podría ser caso de ceceo entre hablantes rurales, periféricos en la ciudad y de clase trabajadora [tranferén

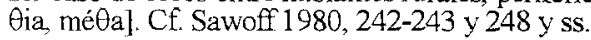

19 Esto es, "el grado de aparición de cada rasgo fonético dialectal en relación con el total de contextos posibles. Es decir, el índice 1 significa que el fenómeno se ha observado en todos los casos analizados. Por el contrario, el índice 0 representaría que el fenómeno no aparece en ninguna ocasión. Por tanto, cualquier cifra entre el 0 y el 1 indica en qué medida los hablantes prefieren, en su uso, el rasgo dialectal frente a lo que sería la realización castellana. Para obtener tales índices, a los rasgos de cada informante se les ha dado una puntuación de forma individual y después se ha obtenido la media para cada uno de los grupos sociolingüísticos considerados" (Carbonero et alii 1992, 18, cf. asimismo 18-21). 
rrección de distinguir s y $z$, al tener el menor índice [de confusión] de los tres grupos» (ibid., 24).

En Granada, F. Salvador (1980) intentó obtener los «índices de utilización del seseo, ceceo y distinción en cada uno de los informantes y sacar el porcentaje medio de la muestra recogida en cada uno de los barrios a los que pertenecían» (ibid., 29). Para ello entrevistó a individuos de ambos sexos de los once barrios (de un total de dieciocho) «más característicos para una investigación lingüística» (ibid., 26 y plano 2). Los sujetos se clasificaron según cuatro niveles educativos y cinco generacionales $(\mathrm{N}=363)^{20}$.

Los resultados de la comparación entre los barrios van en la línea de lo observado por Sawoff en Sevilla. Los barrios tradicionales resultaron ser confundidores de $/ \mathrm{s} / \mathrm{y} / \theta /$, tendiendo los de población mediobaja y trabajadora al seseo con considerables proporciones de ceceo (v. gr., Albaicín: 0.79 y 0.17; Cartuja: 0.44 y 0.30 ), y los de media y medioalta asimismo al seseo con bajos índices de ceceo (Magdalena: 0.60 y 0.03 ; Camino de Ronda: 0.50 y 0.03 ). Los barrios caracterizados por la fuerte inmigración $\mathrm{y}$, por tanto, por las tendencias niveladoras, alcanzaron índices elevados de mantenimiento de la distinción de /s/ : / $\theta /$ (Zaidín: 0.11 y 0.07 de seseo y ceceo, frente a 0.81 de distinción; La Chana: 0.40 y 0.07 , frente a 0.53 ).

En cuanto a las diferencias educativas y generacionales, se observó una fuerte tendencia al mantenimiento de la distinción entre los jóvenes, junto con - dada la estratificación social propia de los barrios- la mejor consideración social del seseo y de la distinción, frente al ceceo. La influencia, según Salvador $(1980,32)$ de «la norma culta castellana que hoy puede ser contrastada con facilidad a través de algunos medios de comunicación (televisión, radio, etc.), unido al hecho de que gran parte de la provincia es distinguidor $a$, han convertido algo que antes era una excepción [la distinción de $\begin{array}{lll}s & y & z\end{array}$, en la tendencia mayoritaria respecto a estos determinados usos de pronunciación. No obstante, el seseo, en algunos barrios de la ciudad, sigue siendo la articulación normal de sus hablantes, mientras que el ceceo se bate en franca retirada». Los porcentajes medios para el conjunto de la ciudad, extraídos de los índices medios de cada barrio, son de 0.41 de seseo, 0.48 de distinción y 0.11 de ceceo.

En Córdoba, Uruburu (1990) estudió el seseo $(\theta>\mathrm{s})$, para comprobar, entre otras cosas, el condicionamiento social de la variación de este fenómeno, patrimonial en la ciudad, pero alterado por diversos motivos, «entre ellos, la

20 Complementariamente, se realizó un sondeo a los empleados del hospital universitario $(\mathrm{N}=25 ; \mathrm{H}=12$ y $\mathrm{M}=13$ ), "con la idea de que los datos de la misma pudieran ser contrastados con el resto ..." (ibid, 32). 
inmigración de personas procedentes del norte de la provincia, en la que se distingue generalmente entre ciceante de $/ \theta /$ y siseante de $/ \mathrm{s} /$, o de otras zonas de España, y el influjo del aparato de la enseñanza, [que] han producido distinción de ciceantes de $/ \theta /$ en distintos porcentajes» (ibid., 125). Centró su atención en veinte informantes, jóvenes estudiantes de enseñanza secundaria y preuniversitaria $(\mathrm{H}=10 ; \mathrm{M}=10)$, que produjeron, en total, 2392 ocurrencias de $/ \theta /$ en estilo casual y formal (ibid., 126)

Las proporciones totales fueron muy ajustadas: 0.51 de seseo $(\theta>\mathrm{s})$, frente a 0.49 de distinción $(/ \theta />[\theta])$, si bien las diferencias genéricas resultan importantes: los hombres alcanzaron el 0.72 de seseo $(0.28$ de $[\theta])$, mientras que las mujeres obtuvieron sólo el 0.27 (0.73 de [ $\theta]$ ). Los sujetos nacidos en Córdoba y cuyos padres nacieron también en la ciudad (ocho en total) subieron el porcentaje de seseo (59.31), hecho debido a la fuerte subida de los cuatro hombres $(89.19 \%)$, frente a las cuatro mujeres, que se mantuvieron en la media general femenina $(28.62 \%)$.

El seseo parece ser, entonces, en Córdoba un rasgo no excesivamente prestigioso en el segmento poblacional estudiado (a falta de investigaciones más generales), habida cuenta de la relación negativa entre este cambio y el número de años de estudios de los padres de los informantes (ibid., 127-128), así como la disminución de la frecuencia del fenómeno observada en el estilo más formal (ibid., 129) ${ }^{21}$.

Recientemente, Caravedo $(1992,642-643)$ ha estudiado la pervivencia de posibles restos de la distinción $/ \mathrm{s} /: / \theta /$ en los Andes peruanos. En dicha zona se encuentran alófonos apicoalveolares estridentes e interdentales mates, como en el español centroseptentrional de la Península Ibérica. La cuestión está en comprobar si su distribución es aleatoria o si se produce alguna especialización alofónica que pudiera hacer pensar en el mantenimiento parcial de la distinción fonológica de $/ \mathrm{s} /: / \theta /$ en una zona aislada unilingüe (Cajamarca) y otra bilingüe (Cuzco).

Los datos provienen de once informantes; los de Cajamarca (3) son sujetos de escasa instrucción formal, de origen rural y de profesión agrícola; los de Cuzco, por el contrario, se dividen en dos grupos; el primero (2), similar al de Cajamarca en su instrucción, es bilingüe, con el quechua como primera lengua, y el segundo (5), de clase media e instrucción superior, también es

21 A ello puede añadirse el dato complementario del comportamiento femenino ante una variable como ésta (un cambio prestigioso o consciente). Dicho comportamiento se ha descrito en mury diversas situaciones como favorecedor de las formas ejemplares o de prestigio global. $\mathrm{Cf}$ W. Labov, "The intersection of sex and social class in the course of linguistic change", Language Variation and Change 2 (1990), 205-254; H. López Morales, "Style variation, sex and linguistic conciousness", in F. Moreno (ed.), Sociolinguistics and stylistic variation, LynX, 3, 1992, Univ. de Valencia - Univ. of Minnesota, Valencia, 1992, 43-54. 
bilingüe, aunque el quechua es segunda lengua (ibid., 644) ${ }^{22}$. Se trata de controlar, naturalmente, la influencia del bilingüismo en el fenómeno estudiado, así como de descartar (o de confirmar) la influencia de la educación formal en las coincidencias entre la realización y la etimología o la grafía de $\mathrm{s}$ y $z$.

Se contabilizaron las realizaciones de $\mid \theta />[\theta]$ y $/ \theta />[\mathrm{s}]$ (complementariamente las de $/ \mathrm{s} />[\mathrm{s}, \theta]$, aun reconociendo que la realización interdental de $/ \mathrm{s} /$ es muy poco frecuente). El cómputo se hizo individualmente, pues «como se trata de explorar la existencia de la oposición o de la dualidad articulatoria, ésta sólo puede observarse de modo adecuado si va referida a cada individuo por separado, pues una contabilización general de las ocurrencias impediría percibir el doble valor de la sibilancia» (ibid., 645). Los resultados (págs. 646-649) muestran la esperada fijación de $[\theta]$ en el contexto canónico $(46 \%, 38 \%, 48 \%$ para los tres informantes de Cajamarca, con porcentajes insignificantes de $/ \mathrm{s} />[\theta]$ ). Los hablantes cuzqueños del grupo iletrado (bilingües, con el quecua como primera lengua) bajan los porcentajes de fijación de $[\theta]$ en el contexto canónico $(28.2 \%$ y $27.7 \%)$, mientras que, de los cinco informantes del grupo de clase media (bilingües, con el español como primera lengua), tres alcanzan porcentajes superiores incluso al grupo unilingüe de Cajamarca $(61.1 \%, 68.7 \%$ y $61.7 \%)$.

Parece claro que la posible conservación de restos de $/ \mathrm{s} /: / 0 /$ no es un fenómeno relacionado con el bilingüismo. Tampoco está conectado con el conocimiento de la ortografía y la influencia de la escuela; el comportamiento general de los grupos estudiados así lo prueba; además, la lectura de textos por parte de los informantes de Caravedo produjo los resultados contrarios: la fijación de $[\theta]$ fue menor que en los textos orales espontáneos (ibid., 649).

La situación andina ${ }^{23}$ muestra «las características de un proceso de desajuste contextual de las sibilantes distintivas, que puede ser significativo si se lo conecta con el problema de la evolución de las sibilantes en español, con la cuestión sincrónica de coexistencia de dos sistemas de oposiciones con distinto grado de variabilidad en la misma lengua, y con las caracteristicas extensivas e intensivas de la variabilidad de las llamadas zonas seseantes» (ibid., 649). Se trata de una situación intermedia entre la total indistinción y distinción caracterizada, en este caso, por el paso gradual de variantes de $/ \theta /$ al espacio o campo de dispersión de /s/ (ibid., 652), que contrasta con la situación americana general y con la costeña peruana en particular, donde la

22 El undécimo informante proviene de la zona central andina (Cerro de Pasco).

23 Que convendría como señala la propia Caravedo, estudiar con mayor detalle. El sujeto del Cerro de Pasco entrevistado complementariamente produjo los siguientes resultados (pág. 649): $\theta$ $>\theta(69.5 \%)$ y $\theta>\mathrm{s}(30.4 \%)$. 
intersección de $/ \mathrm{s} / \mathrm{y} / \theta /$ corresponde ya a la de una total fusión entre las dos unidades, con diferentes variantes dialectales y con diferentes grados de aparición ${ }^{24}$. La indistinción parcial andina se apoya en dos factores esencialmente: $1^{\circ}$ ) el mantenimiento variable de $1 \mathrm{a} / \theta /$ en los contextos canónicos de distinción (veces, bautizo); $2^{\circ}$ ) la marcada diferencia entre la sibilante apical $\left[\mathrm{s}\right.$ ] y la interdental $[\theta]$ en un mismo hablante (ibid., 653) ${ }^{25}$.

\subsection{En Málaga, Ávila (1994) ha estudiado una red social de hablantes del} barrio de Capuchinos en la zona norte del centro de la ciudad $(\mathrm{N}=30 ; \mathrm{H}=12$; $\mathrm{M}=18$ ). El grupo es heterogéneo desde el punto de vista educativo $\mathrm{y}$ generacional, si bien todos los sujetos son de clase trabajadora o media baja. En una primera aproximación a los fenómenos de ceceo y seseo, Ávila contabilizó los trueques de sibilantes $(s>\theta$ y $\theta>\mathrm{s}$ ), frente a los casos de conservación o mantenimiento de su identidad funcional respectiva ( $\mathrm{s}>\mathrm{s}$ y $\theta>$ $\theta$ ). Del mismo modo, estableció los rudimentos del cómputo de la distinción ( $\mathrm{s}$ $>\mathrm{s}+\theta>\theta)$, frente a la indistinción $([\mathrm{s}>\theta]+[\theta>\mathrm{s}])$.

Los resultados, para un total de 4711 ocurrencias de $/ \mathrm{s} / \mathrm{y} / \theta /$, obtenidas en conversaciones dirigidas $y / 0$ en registros inadvertidos con variación estilística temática y conversacional estructural, fueron los siguientes: 0.25 de trueque de $\mathrm{s}>\theta$ (ceceo), frente a 0.75 de mantenimiento de $/ \mathrm{s} / \mathrm{como}[\mathrm{s}] ; 0.31$ de trueque $\operatorname{de} \theta>\mathrm{s}$ (seseo), frente al 0.69 de mantenimiento de $/ \theta /$ como $[\theta] ; 0.70$ de distinción, frente a 0.30 de indistinción ${ }^{26}$. El seseo resultó un fenómeno

24 Galeote $(1988,22-70)$, en su detallada investigación en el Treviño malagueño-granadinocordobés de Iznájar, Villanueva de Tapia y Venta de Santa Bárbara, extrajo datos de 75 informantes de ambos sexos, divididos en cinco generaciones y separados asimismo por su dedicación a labores agrícolas o urbanas. Sus resultados se centran en: $1^{\circ}$ ) el establecimiento de las áreas geográficas de seseo, ceceo y distinción de /s/ : $10 /$ y sus pautas de realización correspondientes; $2^{\circ}$ ) la fijación de las pautas de seseo con [s] coronal, seseo con [s] predorsal y su distribución geográfica y variación sociolingüística en la citada zona de transición; $3^{\circ}$ ) la determinación de los grados de polimorfismo en la realización de $/ \mathrm{s}$ y $/ \theta /$ y su relación con las diferencias generacionales y genéricas.

25 "No es concebible, pues, que en este estadio de indiferenciación total, los hablantes conserven dos tipos articulatorios tan definidos como la apical frente a la interdental, distribuidos además en proporciones significativas de acuerdo con sus contextos originarios" (Caravedo 1992, 654). La cuestión es si se aplica asimismo la proposición contraria; esto es, si es posible la distinción $/ \mathrm{s} /: \theta /$ mediante alófonos, respectivamente, estridentes y mates, pero todos dentales. Cf., más arriba, la nota 13 .

26 Los resultados varían al incluir los estilos gráficos obtenidos por medio de pruebas especificas; número total de ocurrencias de $/ \mathrm{s} / \mathrm{y} / \theta /=6396$ : trueque de $/ \theta />\mathrm{s}=0.23$; trueque de $/ \mathrm{s}>\theta=0.23$. La variación genérica produjo los siguientes resultados: 0.12 frente a 0.33 , respectivamente, para hombres y mujeres en el seseo, y 0.42 frente a 0.10 en el ceceo. Como se ve, al introducir los estilos gráficos, descendió el porcentaje de ambos cambios, si bien fue superior el descenso del seseo. En cuanto a las diferencias genéricas, bajó considerablemente el trueque de $\theta>\mathrm{s}$ en las mujeres (en beneficio de la distinción), y subió algo en los hombres (en perjuicio del ceceo): el cambio de $s>\theta$ bajó en los hombres, paralelamente a la subida del seseo 
claramente femenino (el 0.50 de las z etimológicas se convirtió en [s], frente al $0.11 \mathrm{en}$ el caso de los hombres), mientras que el ceceo se inclinaba del lado de los hombres ( 0.45 de $s>\theta$, frente a 0.11 para las mujeres). Se constató asimismo la relación positiva entre el nivel de instrucción formal y los porcentajes de distinción, muy elevados, si los comparamos con los comentados arriba para otras ciudades $(0.52,0.67,0.70$ y 0.91 , respectivamente, para los sujetos sin titulación, con estudios primarios, medios y universitarios $)^{27}$ y la relación negativa entre la edad y la frecuencia de mantenimiento de $/ \mathrm{s} / \mathrm{y} / \theta /$ $\left(0.92,0.76,0.72\right.$ y 0.54 , respectivamente, para los hablantes de la $\mathrm{I}^{\mathrm{a}}, \mathrm{II}^{\mathrm{a}}, \mathrm{II}^{\mathrm{a}} \mathrm{y}$ IV generación). Por el contrario, no se constataron diferencias significativas en la relación entre los trueques de sibilantes y las desigualdades y diferencias sociales distintas del género.

Los resultados del análisis probabilístico de los mismos datos, a partir del modelo multinomial objeto del presente trabajo ( $\mathrm{y}$ basado, como en el caso del estudio de Caravedo, en el comportamiento individual), no indicaron contradicciones importantes con respecto a la variación de las frecuencias observadas, pero redujeron notablemente, desde el punto de vista de la interpretación, la importancia de la distinción de $/ \mathrm{s} /: / \theta /$ en la comunidad local estudiada y, consecuentemente, corrigieron la de la indistinción, sea seseante 0 ceceante. La probabilidad media de distinción es de .47 (frente al 0.69 de $\mathrm{s}>\mathrm{s}$ $+\theta>\theta$ arriba consignado); la de seseo es de .35 (frente al 0.31 de $\theta>\mathrm{s}$ ) y la de ceceo es de .26 (frente al $0.25 \mathrm{de} \mathrm{s}>\theta$ ). La variación sociolingǘstica ofrece los siguientes datos: se confirma la bipolarización genérica para el seseo (hombres, probabilidad media: .15; mujeres: .49) y para el ceceo (hombres, probabilidad media: .49; mujeres: .11); la estratificación educacional y las distinciones generacionales presentan diferencias significativas en lo concerniente a la probabilidad de distinción (correspondientemente, indistinción), pero no en lo tocante al seseo o al ceceo: probabilidad media de distinción de $.19, .50, .70$, respectivamente, para los individuos con menos de siete años de estudios, entre siete y catorce, y más de catorce, y de $.55, .45, .18$, para los individuos de edades entre los 20-34, 35-55 y más de 55, respectivamente ${ }^{28}$.

comentada arriba, y se mantuvo entre las mujeres. Los datos generales indicados excluyen las realizaciones intermedias $\left(\theta^{\mathrm{s}}, \mathrm{s}^{\theta}\right)$, cuya frecuencia es muy bajà: $0.01(<\mathrm{s})$ y $0.03(<\theta)$.

27 La estratificación por clases sociales (construidas como una variable de status a partir de la educación. la profesión y la renta) ofreció un contraste entre el 0.64 para la clase trabajadora y trabajadora baja ( $0-5$ en una escala de $0-9)$ y 0.85 para la clase media baja (6-8 en la misma escala).

z8 Véase asimismo Villena y Requena 1994. 
2.4. En el análisis de regresión logística realizado con Goldvarb $2.0^{29} \mathrm{del}$ cambio de s etimológica $>[\theta]$ resultaron significativos los grupos de factores siguientes y por el siguiente orden: sexo, edad, clase social, estilo. Las diferencias debidas al entorno lingüístico (presencia de [s] o [ $\theta$ ] en la palabra) no son significativas y las pequeñas fluctuaciones observadas se deben al azar (frente al caso del cambio $\theta>\mathrm{s}$ ). La probabilidad input $\left(\mathrm{P}_{0}=.166\right)$ es baja, dado que el valor de aplicación considerado es únicamente $\mathrm{s}>\theta$, tomándose como valor de no aplicación los casos de $s>s$, muy abundantes. La probabilidad media de ceceo $(\mathrm{P}(\mathrm{c}))$ en el modelo probabilístico basado en multinomiales asciende, por efecto de la corrección inherente al mismo, a $\mathrm{P}=$ .259 .

\begin{tabular}{|c|c|c|c|}
\hline \hline Sexo & Estilo & Edad & Clase social \\
\hline H. .79 & Oral: .54 & IV $^{\mathrm{a}}: .77$ & $(0-5): .54$ \\
\hline
\end{tabular}

TABLA n ${ }^{\circ} 1$. Análisis Goldvarb de $s>\theta$ en Málaga. Datos del Barrio de Capuchinos. $\mathrm{N}=30 ; \mathrm{n}^{\circ}$ de ocurrencias de $/ \mathrm{s} /=4072$; Estilo: oral $/$ gráfico; clase social ( $0-9$, profesión, educación y renta). $P_{0}: 166$.

En el análisis efectuado sobre el cambio de $\mathrm{z}$ etimológica $>$ [s], para un total de 2324 ocurrencias de $/ \theta /$, resultaron significativos los siguientes grupos de factores y en el siguiente orden: sexo, edad, clase social, estilo y entorno lingüístico ${ }^{30}$. Las diferencias debidas a la presencia de /s/o / $/$ / en la palabra constituyen una variable condicionante de la variación $z>s$. La s-precedente

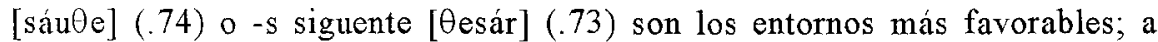
continuación la $\theta$-precedente $[\theta$ eré $\theta$ a]; el resto tiene efecto negativo (ausencia de $/ \mathrm{s} / \mathrm{o} / \theta /, / \theta /$ siguiente, palatales, etc.). Como se ve, la tendencia es, cuando aparece, hacia la igualación de /s/ en la palabra: [sáuse], [sesár]; si hay $/ \theta /$ precedente, entonces se tiende a la disimilación: [ $\theta$ erésa]. La probabilidad input (.163) es, como en el caso anterior, baja, dado que el valor de aplicación considerado es únicamente $\theta>\mathrm{s}$, tomándose como valor de no aplicación los casos de $\theta>\theta$. La probabilidad media de seseo $(\mathrm{P}(\mathrm{s}))$ en el modelo

29 Vid. D. Sankoff y D. Rand, Golduarb. Version 2.0. A Variable Rule Application for the Macintosh ${ }^{\text {Th }}$, Centre de Recherches Mathématiques, Université de Montréal, 1990.

30 Los resultados del análisis de los datos extraídos de los estilos orales exclusivamente ( 1280 apariciones de $z$ etimológica) produjeron la selección, como grupos de factores significativos en la explicación de la variación de $z>s$, de los entornos fónicos previos y posteriores en la palabra y en las palabras adyacentes, de entre un total de grupos de factores internos, sociales y estilísticos de veintiocho $\left(\mathrm{P}_{0}=.234\right.$; mujer .72; estudios medios .70 , sin estudios .67; entorno: palabra $-\mathrm{s} .64$, $\theta \sim .65,-s .64$; secuencia: $s-.66 ; s / \theta-.66,-\theta .51$ ). Por lo que se refiere al proceso de $s>\theta$, el análisis de 3387 tokens $s$ con $s$ etimológica produjo los resultados siguientes, a partir del mismo esquema de partida: $P_{0}=.147$; hombre $.90 ; I^{2}$ generación .85 . III ${ }^{2} .62$; sin educación .67 , primaria, media .51; estulo informal .53 ; entorno $s / \theta-.69, \mathrm{~s}-.57$. 
probabilístico basado en multinomiales asciende a $P=.3473$. La probabilidad de mantenimiento $\left(\mathrm{s}>\mathrm{s}\right.$ y $\mathrm{z}>\theta$ ) se obtiene en los dos análisis como $1-\mathrm{P}_{0}$ (siendo 1 equivalente a la $\mathrm{P}_{0}$ resultante en cada uno de los análisis de los trueques de $\mathrm{s}>\theta$ y de $\mathrm{z}>\mathrm{s}$ ), resultando, entonces, muy elevada (.834 y .837 , respectivamente). Sin embargo, la probabilidad de distinción de $/ \mathrm{s} /: / \theta /$ en el modelo probabilístico multinomial es de .4654 .

\begin{tabular}{|c|c|c|c|c||}
\hline Sexo & Entorno lingüístico & Estilo & Edad & $\begin{array}{c}\text { Clase } \\
\text { Social }\end{array}$ \\
\hline $\mathrm{M}: .66$ & $\mathrm{~s}-: .74$ & & $\mathrm{IV}^{\mathrm{a}}: .60$ & \\
& $-\mathrm{s}: .73$ & Oral: .61 & $\mathrm{II}^{\mathrm{a}}: .59$ & $(0-5): .60$ \\
\hline
\end{tabular}

Tabla $n^{\circ} 2$. Análisis Goldvarb de $\mathrm{z}>\mathrm{s}$ en Málaga. Datos del Barrio de Capuchinos. $\mathrm{N}=30 ; \mathrm{n}^{\circ}$ de ocurrencias de $/ \theta /=2324$; Entorno lingüístico: $\mathrm{s}-$ precedente, -s previa, $\theta$-previa en la misma palabra; Estilo: oral / gráfico; clase social (0-9, profesión, educación y renta). P0: 163.

2.5. Lo que todos estos estudios -salvo el de Ávila- tienen en común es la consideración parcial (trueques) del conjunto de los fenómenos, como procedimiento para realizar los cómputos de seseo $(\theta>\mathrm{s})$ y de ceceo $(\mathrm{s}>\theta)$, así como la omisión o el desconocimiento del cortocircuito señalado por Sawoff $(\theta>\theta$ como distinción o como ceceo y $\mathrm{s}>\mathrm{s}$ como distinción o como seseo). El establecimiento de los medios para solucionar este problema es el objetivo de este trabajo.

La cuestión radica básicamente en la adopción de una actitud razonablemente realista al considerar las pautas individuales de realización de / $\theta$ s/, más allá del mero recuento de la frecuencia de los trueques o del mantenimiento de $/ \mathrm{s} /$ y $/ \theta /$. Los modelos de representación de las pautas de seseo o de ceceo han de incluir no sólo los trueques correspondientes $(\theta>\mathrm{s} ; \mathrm{s}>$ $\theta$ ), sino asimismo la estimación del porcentaje de casos de realización de $/ \mathrm{s} /$ como [s] y de $/ \theta /$ como $[\theta]$ que han de sumarse a dichos trueques en el cálculo de la probabilidad seseante o ceceante. Tal estimación depende únicamente de la relación entre la etimología (esto es, la realización en los contextos canónicos) y la realización efectiva, así como de la unidad de comportamiento lingüistico que constituye el individuo.

La diferencia entre un modelo frecuencial -basado en las proporciones de los trueques entre $/ \mathrm{s} / \mathrm{y} / \theta /$ en relación con el total, y en la suma de los aciertos o realizaciones de $/ \mathrm{s} /$ y $/ \theta /$ en sus contextos canónicos- y un modelo estocástico individual o idiolectal debería ser captada intuitivamente, al 
comparar los datos de manera conjunta, tal y como se hace en la tabla $n^{\circ} 3$. En ella se han seleccionado sujetos seseantes (M.C.) y ceceantes (A.T.); sujetos con porcentajes intermedios (C.O. y L.A.), y sujetos con porcentajes muy bajos o inexistentes de seseo y ceceo (A.R.), todos ellos del grupo de individuos estudiados por Ávila (1994). En todos los casos, las diferencias entre el modelo frecuencial $y$ el probabilístico son evidentes. Ahora bien, si de la pauta individual se pasa a los promedios, la diferencia entre el manejo de los datos frecuenciales brutos y los procedentes de la estimación probabilística son claros únicamente en el caso de la distinción / indistinción $(70.4 \%$, frente a $.47, \pm .35$ ). En lo tocante al seseo / ceceo, sin embargo, dada la dispersión existente debida a la bipolarización genérica del comportamiento con respecto a esta variable (probabilidad media de ceceo $=.26 \pm .35$; probabilidad media de seseo $=.35 \pm .39$ ), las diferencias individuales mostradas por el modelo probabilístico se difuminan. En sí mismas demuestran, a la vez, la raíz de la poca transparencia de las probabilidades medias empleadas con finalidad comparativa. Éstas no se presentan ni siquiera claras en los grupos de hablantes en los que las frecuencias de trueques son más altas.

Pseseo $\quad z>s(\%) \quad$ Pceceo $\quad s>\theta(\%) \quad$ Pdistin $\quad s>s$

\begin{tabular}{|c|c|c|c|c|c|c|}
\hline & & & & & & $>\theta(\%$ \\
\hline $\begin{array}{l}\text { Prob } \\
\text { media }\end{array}$ & .35 & 31.5 & .26 & 25.5 & .47 & 70.4 \\
\hline $\begin{array}{l}\text { Pmedia } \\
\text { sin } \\
\text { estudios }\end{array}$ & $\begin{array}{l}\mathrm{H}: .09 \\
\mathrm{M}: .73\end{array}$ & $\begin{array}{l}\mathrm{H}: 05.0 \\
\mathrm{M}: 72.3\end{array}$ & $\begin{array}{l}H: .85 \\
M: .14\end{array}$ & $\begin{array}{l}\mathrm{H}: 85.1 \\
\mathrm{M}: 12.1\end{array}$ & - & - \\
\hline $\begin{array}{l}\text { Pmedia } \\
\text { S.E. y } \\
\text { Primarios }\end{array}$ & $\begin{array}{l}H: .17 \\
M: .50\end{array}$ & $\begin{array}{c}\mathrm{H}: 8.2 \\
\mathrm{M}: 59.3\end{array}$ & $\begin{array}{l}\mathrm{H}: .72 \\
\mathrm{M}: .09\end{array}$ & $\begin{array}{l}\mathrm{H}: 73.1 \\
\mathrm{M}: 16.6\end{array}$ & - & - \\
\hline $30 \mathrm{MC}$ & .98 & 39.0 & .00 & 00.0 & .22 & 61.0 \\
\hline $3 \mathrm{AT}$ & .00 & 00.0 & .74 & 44.0 & .12 & 56.0 \\
\hline $13 \mathrm{CO}$ & .11 & 06.0 & .64 & 26.0 & .34 & 67.0 \\
\hline $8 \mathrm{AR}$ & .02 & 01.0 & .21 & 07.0 & .83 & 91.0 \\
\hline $21 \mathrm{LA}$ & .44 & 13.0 & .11 & 08.0 & .45 & 80.0 \\
\hline
\end{tabular}

TABLA n ${ }^{\circ} 3$. Frecuencias y probabilidades individuales de seseo, ceceo y distinción comparadas con ias probabilidades medias y las frecuencias totales. Datos de Capuchinos (Málaga).

2.6. La comparación de los datos disponibles es difícil, dada la heterogeneidad de los procedimientos empleados en los distintos estudios, así como la falta, a reces, de la necesaria exposición explícita de los cálculos efectuados. En todo 
caso, lo único que se pretende aquí es ofrecer una imagen orientativa de la situación. En las tablas siguientes, se han separado los resultados en función de si se han efectuado cálculos individuales de seseo, ceceo y distinción, a los que con posterioridad se les han calculado porcentajes o indices medios, o, por el contrario, si se ha trabajado directamente con las frecuencias de las realizaciones de los sonidos.

Hay asimismo una diferencia importante. En la primera (tabla $n^{0} 4$ ), se consideran las proporciones e índices sobre el total ( $\mathrm{s}+\mathrm{z}$ etimológicas), de modo que seseo + ceceo + distinción $=1$. En la segunda $\left(\operatorname{tabla} n^{\circ} 5\right)$, por el contrario, se consideran separadamente los cambios $\mathrm{z}>\mathrm{s}$ y $\mathrm{s}>\theta$, de modo que las proporciones se dan sobre los parciales de $z$ etimológica y $s$ etimológica (cf., más adelante, párr. 4).

En la tabla $n^{\circ} 4$ se incluyen los índices porcentuales medios (Salvador 1980) y los índices de aceptación (Carbonero), calculados individualmente, con expresión final de promedios:

\begin{tabular}{||l||c|c|c||}
\hline \hline SEVILLA & SESEO & CECEO & $\begin{array}{c}\text { DISTIN- } \\
\text { CIÓN }\end{array}$ \\
\hline JEREZ & 0.87 & 0.06 & 0.07 \\
\hline GRANADA & 0.44 & 0.47 & 0.09 \\
\hline \hline
\end{tabular}

TABLA $n^{\circ} 4$. Índices porcentuales de seseo, ceceo y distinción de $s / \theta$ en el español andaluz. Fuente: Sevilla (Carbonero 1982, 1985); Jerez (Carbonero et alii 1992); Granada (Salvador 1980).

En la tabla $n^{\circ} 5$ se han agrupado con fines comparativos los datos ofrecidos en aquellos estudios en los que se han calculado las frecuencias relativas de los cambios de $s>\theta$ y de $\theta>s$ (junto con los mantenimientos correspondientes), de modo que aquéllas deben ser interpretadas, respectivamente, en relación al total de casos de $\mathrm{s}$ y de $\mathrm{z}$ etimológicas. 


\begin{tabular}{||l|c|c|c|c||}
\multicolumn{2}{|c|}{ z etimológica } & \multicolumn{2}{c||}{ s etimológica } \\
\hline \hline Realización & {$[\mathrm{s}]$} & {$[\theta]$} & {$[\mathrm{s}]$} & {$[\theta]$} \\
\hline Sevilla & & & & - \\
\hline Córdoba $^{* *}$ & 0.66 & 0.34 & - & - \\
& 0.54 & 0.45 & & - \\
\hline Cajamarca $^{* * *}$ & 0.51 & 0.49 & & 0.10 \\
\hline Cuzco $^{* * * *}$ & 0.53 & 0.46 & 0.90 & - \\
\hline Capuchinos & 0.28 & 0.72 & - & - \\
(Málaga) & 0.38 & 0.62 & - & 0.25 \\
$* * * * *$ & 0.31 & 0.69 & 0.75 & 0.26 \\
\hline
\end{tabular}

TABLA ${ }^{\circ} 5$. Porcentajes de las realizaciones de $/ \theta \mathrm{s} /$ en función de su origen etimológico. Fuente: Sevilla (Sawoff 1980); Córdoba (Uruburu 1990);

Cajamarca y Cuzco (Caravedo 1992); Málaga (Ávíla 1994).

* La primera fila corresponde a las respuestas de los hablantes de menor prestigio profesional; la segunda a los de mayor prestigio.

** La muestra considerada es de 20 individuos ( $<29$ años)

*** Datos frecuenciales individuales (clase popular, escasa cultura, primera generación).

**** Id. (en la fila de arriba, datos de un hablante de clase popular y primera generación; en la de abajo, hablante de clase media).

****** Porcentajes totales en la fila de arriba; en la de abajo, probabilidades medias.

\section{VARIABLES}

El estudio de la variación del fonema $/ \theta^{s} /(<\dot{s}, \dot{z}, \hat{s}, \hat{z})$ en una comunidad como la malagueña es ciertamente difícil, ya que en ella coexisten como normas ideales las del ceceo, seseo y distinción, presentándose en los individuos de un modo bastante irregular y alternando en su utilización unas y otras de una manera aparentemente aleatoria.

Las realizaciones consideradas aquí son:

$$
\begin{array}{ll}
\mathrm{s}>[\mathrm{s}] & \mathrm{s}>[\theta] \\
\mathrm{z}>[\mathrm{s}] & \mathrm{z}>[\theta],
\end{array}
$$


y los datos que se utilizarán para el desarrollo de este modelo (y los únicos observables en relación con el objetivo planteado) son las frecuencias absolutas de estas distintas realizaciones en cada individuo.

Generalmente, este recuento de frecuencias ha constituido el procedimiento normal para la estimación del grado de ceceo y seseo frente a distinción o mantenimiento, tal y como se ha analizado arriba (párr. 2). Sin embargo, este mero recuento presenta un defecto estructural fundamental, al considerarse el problema desde el punto de vista de la norma de pronunciación: cuando una persona sesea, tiende a convertir la $[\theta]$ etimológica en [s], pero también realiza la $|\mathrm{s}|$ etimológica como $[\mathrm{s}]$, y en este último caso no porque la sitúe en su espacio funcional canónico, distinguiendo, sino debido a que, por su norma, tiende a transformar en $[s]$ todas sus realizaciones. Este comportamiento, que también se registra para la $|\theta|$ etimológica con el ceceo, no es tomado en cuenta por el modelo basado en los recuentos, restándole así exactitud desde un punto de vista estadístico (Sawoff, 1980, 242-243).

El enfoque que parece más adecuado es aquel en el que se considera que seseo, ceceo y distinción son normas ideales a las que el hablante tiende, pero que, en realidad, mezcla de una manera aleatoria y desordenada en ciertas proporciones en un continuo s $\theta$ (Carbonero 1982; Sawoff 1980; Villena y Requena 1994), con aproximaciones relativas a los polos.

Estos hechos obligan a buscar un modelo probabilístico que permita, a partir de las frecuencias observadas, localizar a cada individuo, de una manera efectiva, en ese continuo s

Para poder llevar a cabo el estudio que se presenta en este trabajo, hay que hacer varias suposiciones:

$\left.1^{\mathrm{a}}\right)$ La pauta de pronunciación de $/ \theta^{\mathrm{s} /}$ es independiente del entorno lingüístico en el que se estudia cada realización.

$2^{2}$ ) Cada realización es independiente de las restantes, y sólo depende de la pauta de conducta del hablante.

Estas dos suposiciones permitirán establecer un sistema para identificar al hablante desde el punto de vista de su comportamiento lingüístico, independientemente del entorno de los elementos que componen la muestra analizada (suposición $1^{a}$ ); en dicho sistema cada elemento de la muestra será independiente de los demás elementos (suposición $2^{\mathrm{a}}$ ). Esta última condición permite construir un modelo multinomial, cuyos resultados han de ser uniformemente aplicables en cualquier momento de la conversación, con independencia del contexto lingüístico.

La concepción primera que permite llevar a cabo la identificación del individuo, desde el punto de vista de la pronunciación de $/ \theta^{\mathrm{S}} /$, es que cada uno mezcla de manera arbitraria los tres modelos ideales de seseo, ceceo y 
distinción en unas ciertas proporciones aproximadamente constantes. La pregunta crucial es ¿cuáles son estas proporciones?

\section{CONSTRUCCIÓN DE LOS MODELOS}

\subsection{Modelo de Ceceo-Seseo.}

El primer paso del desarrollo consiste en especificar de manera explícita y funcional qué se entiende por norma ideal de seseo, ceceo y distinción:

- Un seseante prototípico se supone una persona que únicamente lleva a cabo las realizaciones $\mathrm{s}>[\mathrm{s}]$ y $\mathrm{z}>$ [s], lo cual se podría expresar mediante probabilidades condicionadas de la realización frente a la etimología, como:

\begin{tabular}{|c|c|c|c|}
\hline P[ Realización & | etimología] & Realiz & \\
\hline & Etimología & [s] & {$[\theta]$} \\
\hline & $s$ & 1 & 0 \\
\hline & $z$ & 1 & 0 \\
\hline
\end{tabular}

- Un ceceante prototípico, por el contrario, se supone que sólo llevará a cabo realizaciones $\mathrm{z}>[\theta]$ y $\mathrm{s}>[\theta]$, con lo que sus probabilidades condicionadas son:

\begin{tabular}{c|c|c|}
$\mathrm{P}[$ Realización $\mid$ etimología] & \multicolumn{2}{|c|}{ Realización } \\
\cline { 2 - 3 } Etimología & {$[\mathrm{s}]$} & {$[\theta]$} \\
\hline $\mathrm{s}$ & 0 & 1 \\
\hline $\mathrm{z}$ & 0 & 1 \\
\hline
\end{tabular}

- Un distinguidor prototípico sólo llevará a cabo realizaciones $s>$ $[\mathrm{s}] \mathrm{y} \mathrm{z}>[\theta]$ :

$\mathrm{P}[$ Realización / etimología]
\begin{tabular}{c|c|c|}
\hline Etimología & {$[\mathrm{s}]$} & {$[\theta]$} \\
\hline $\mathrm{s}$ & 1 & 0 \\
\hline $\mathrm{z}$ & 0 & 1 \\
\hline
\end{tabular}

Se manifiestan así claramente, desde el punto de vista formal, las normas ideales de ceceo, seseo y distinción, que se denotarán a partir de ahora como $\mathrm{C}$, $\mathrm{S}$ y $\mathrm{B}$, respectivamente, y cuyo comportamiento se puede ver en el gráfico 1 . 
S

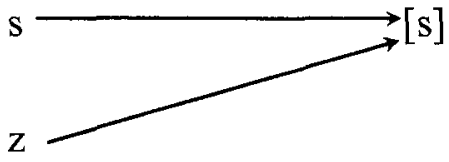

$\mathrm{C}$
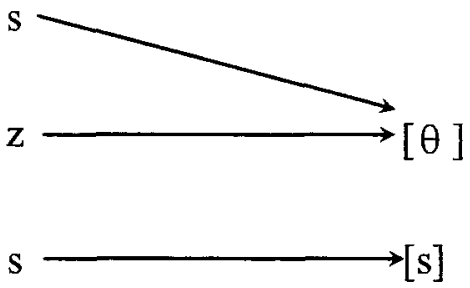

B

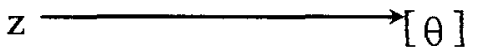

Gráfico 1: Normas ideales de pronunciación de $/ \theta^{\mathrm{S} /}$

Los hablantes se relacionan variablemente con estos tres modelos en función de múltiples factores (Ávila 1944; Villena y Requena 1994). El problema aquí consiste en encontrar cuál es el mejor modo de cuantificar esta relación. Cabe imaginar que los individuos alternan en su comportamiento

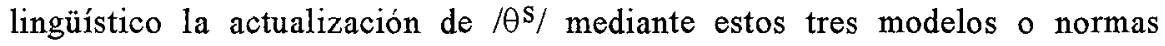
ideales. El cuestión residiría, entonces, en estimar, por medio del estudio de las frecuencias absolutas, en qué proporción de casos utilizan los individuos cada modelo. A cada una de estas proporciones se las denotará $P S, P C$ y $P B$ para los modelos de seseo, ceceo y distinción, respectivamente. El modelo queda como se muestra en el gráfico 2: 
Hablante
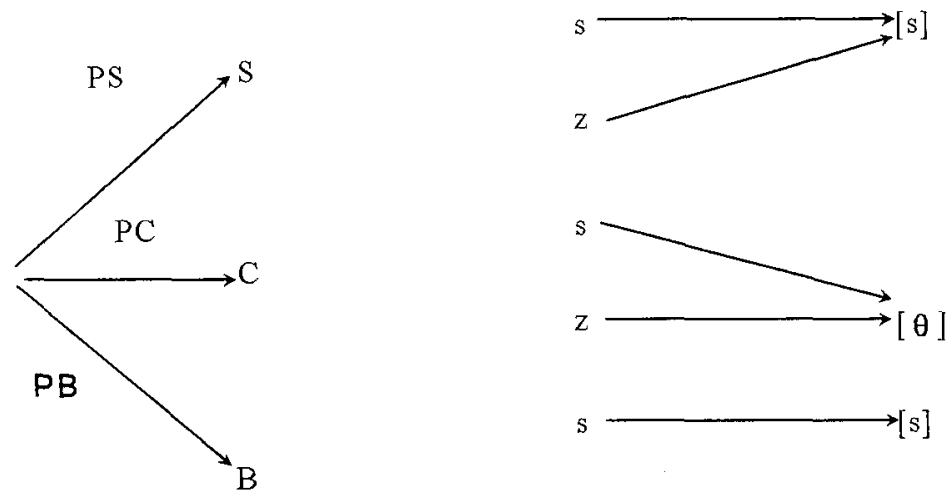

s

Z

Gráfico 2: Modelo general de realización individual de $/ \theta^{\mathrm{S}} /$ y pautas de comportamiento lingüístico implícito

Como se puede observar, ante una realización virtual, el individuo escoge con una cierta probabilidad cada uno de los modelos, y actúa consecuentemente. Por lo tanto, el hablante quedaría caracterizado, desde el punto de vista fonológico, si se estiman $P S, P C$ y $P B$.

Para la estimación de $P S, P C$ y $P B$, se dispone como punto de partida del número de realizaciones de cada tipo $(\mathrm{s}>[\mathrm{s}], \mathrm{s}>[\theta], \mathrm{z}>[\mathrm{s}], \mathrm{z}>[\theta])$ para cada individuo de la muestra, por lo que nuestro próximo objetivo es relacionar las probabilidades $P S, P C$ y $P B$ con estas frecuencias observadas.

A estas frecuencias se las denotará por:

- Nsas o la frecuencia absoluta de la realización $s>[s]$.

- Nsaz o la frecuencia absoluta de la realización $\mathrm{s}>[\theta]$.

- Nzas o la frecuencia absoluta de la realización $z>[s]$.

- Nzaz o la frecuencia absoluta de la realización $z>[\theta]$.

Para el desarrollo del modelo, es necesario que se supongan conocidas, en principio, las probabilidades de realizar $P[s>[s]], P[s>[\theta]], P[z>[s]]$ y $P[z>[\theta]]$, construyendo a continuación un modelo probabilístico que nos permita encontrar la probabilidad de obtener en una muestra individual $N$ sas $=\alpha, N s a z=\beta, N z a s=\gamma$ y $N z a z=\delta$, considerando cada realización independiente de las restantes, y posteriormente estimar los valores de los parámetros que intervienen en la distribución $(P S, P C$ y $P B)$ a partir de los valores muestrales. La probabilidad de obtener en una muestra 
individual $N s a s=\alpha, N s a z=\beta, N z a s=\gamma$ y Nzaz $=\delta$ viene dada por el producto de las probabilidades asociadas a cada una de las realizaciones, es decir, el producto de $\alpha$ probabilidades $P[s>[s]], \beta$ probabilidades $P[s>$ $[\theta]], \gamma$ probabilidades $P[z>[\theta]]$ y $\delta$ probabilidades $P[z>[\theta]]$ :

$$
\begin{gathered}
P[s>[s]] \cdots P[s>[s]] \cdot P[s>[\theta]] \cdots P[s>[\theta]] . \\
\quad P[z>[s]] \cdots P[z>[s]] \cdot P[z>[\theta]] \cdots P[z>[\theta]] .
\end{gathered}
$$

De ahí, conociendo que el producto de $n$ términos iguales, $a$, es equivalente a $\mathrm{a}^{\mathrm{n}}$, se obtendría:

$$
\begin{gathered}
P[s>[s]]^{\alpha} \cdots P[s>[s]]=P[s>[s]]^{a}, \\
P[s>[\theta]]^{\beta} \cdots P[s>[\theta]]=P[s>[\theta]]^{\beta}, \\
P[z>[s]] \stackrel{\gamma}{*} P[z>[s]]=P[z>[s]]^{\gamma}, \\
P[z>[\theta]]^{\infty} \cdots P[z>[\theta]]=P[z>[\theta]]^{\delta},
\end{gathered}
$$

de donde se extrae, para la expresión de la probabilidad:

$$
P[s>[s]]^{\alpha} P[s>[\theta]]^{\beta} P[z>[s]]^{\gamma} P[z>[\theta]]^{\delta},
$$

para cada una de las posibles ordenaciones de los $\alpha+\beta+\gamma+\delta$ elementos de la muestra. Así, se ha conseguido la expresión de la probabilidad de obtener cierto número de realizaciones de cada tipo en una muestra, para cada una de las posibles ordenaciones de todas las realizaciones tomadas en consideración. Lo que se busca es que la estimación dependa únicamente del número de realizaciones de cada tipo llevadas a cabo, sin que tenga importancia el orden en que se produjeron, ya que para el estudio del seseo-ceceo este dato es superfluo. Por esto, se calcula la probabilidad de obtener $N s a s=\alpha, N s a z=\beta$, $N z a s=\gamma$ y Nzaz $=\delta$, para cada una de las posibles ordenaciones, y se suman todas, para de esta manera dar con la probabilidad de obtener esta configuración en los resultados muestrales, independientemente de la ordenación de la muestra. Puesto que la probabilidad de obtener cada una de las ordenaciones viene dada por la misma expresión:

$$
P[s>[s]]^{\alpha} P[s>[\theta]]^{\beta} P[z>[s]]^{\gamma} P[z>[\theta]]^{\delta},
$$

tan sólo resta calcular cuántas posibles configuraciones de este tipo hay

El número de posibles ordenaciones de $n$ elementos distintos es todas las formas en las que se pueden ordenar los $n$ elementos sin que se repita ninguno, y viene dada por el número de elementos que se pueden colocar en el primer lugar (cualquiera de los $n$ elementos), $n$, multiplicado por el número de elementos que se pueden colocar en segundo lugar (todos menos el que se ha colocado en el primero), $n-1$, y así sucesivamente, $n-2, n-3, \ldots$, hasta que sólo 
quede un elemento para colocar en el último lugar, 1. Por lo tanto, el número de posibles ordenaciones de $\mathrm{n}$ elementos distintos viene dado por la expresión:

$$
n \cdot(n-l) \cdot(n-2) \cdot \ldots \cdot 3 \cdot 2 \cdot 1 \text {, }
$$

valor al que se denomina factorial de $n, \mathrm{y}$ que se denota por $n !$ :

$$
n !=n \cdot(n-1) \cdot(n-2) \cdot \ldots \cdot 3 \cdot 2 \cdot 1 \text {. }
$$

El número de posibles ordenaciones de $n=\alpha+\beta+\gamma+\delta$, elementos, donde hay $\alpha, \beta, \gamma$ y $\delta$ elementos iguales (en este caso el número de veces que se dan por individuo los casos de $s>[s], s>[\theta], z>[s]$ y $z>[\theta]$ ), es el número de permutaciones totales de $\operatorname{los} n$ elementos, eliminando configuraciones que se obtienen intercambiando términos iguales. Dada una configuración cualquiera, el número de configuraciones equivalentes que se puede obtener intercambiando los $\alpha$ elementos iguales viene dado, según ya se ha visto, por la expresión $\alpha$ !, al intercambiar los $\beta$ elementos iguales, $\beta$ !, y de manera equivalente, $\gamma$ ! y $\delta$. En consecuencia, se obtiene que el número de configuraciones equivalentes en las $n$ ! configuraciones dadas por todas las posibles ordenaciones de los $n$ elementos, viene dado por la expresión $\alpha ! \cdot \beta ! \cdot \gamma ! \cdot \delta !$, con lo que el número de posibles ordenaciones de los $n=\alpha+\beta+\gamma$ $+\delta$, donde se repiten $\alpha, \beta, \gamma$ y $\delta$ elementos, viene dado por la expresión:

$$
\frac{(\alpha+\beta+\gamma+\delta) !}{\alpha ! \cdot \beta ! \cdot \gamma ! \cdot \delta !}
$$

que se denota por $P R_{\alpha+\beta+\gamma+\delta}^{\alpha, p, \gamma, \alpha}$. Puesto que la probabilidad encontrada anteriormente:

$$
P[s>[s]]^{\alpha} P[s>[\theta]]^{\beta} P[z>[s]]^{\gamma} P[z>[\theta]]^{\delta}
$$

estaba aplicada a cada caso particular de las posibles ordenaciones de $N s a s=$ $\alpha, N s a z=\beta, N z a s=\gamma$ y $N z a z=\delta$, la expresión de la probabilidad de obtener $N_{s a s}=\alpha, N s a z=\beta, N z a s=\gamma$ y $N z a z=\delta$, independientemente del orden de ocurrencia, vendrá dada por la suma de todas esas probabilidades individuales, lo cual, al final, se puede expresar como:

de donde:

$$
P R_{\alpha+\beta+\gamma+\delta}^{\alpha, \beta, \gamma, \delta} P[s>[s]]^{\alpha} P[s>[\theta]]^{\beta} P[z>[s]]^{\gamma} P[z>[\theta]]^{\delta},
$$

$$
\begin{aligned}
P[N \text { sas }=\alpha & , N s a z=\beta, N z a s=\gamma, N z a z=\delta]= \\
& =\frac{(\alpha+\beta+\gamma+\delta) !}{\alpha ! \beta ! \gamma ! \delta !} P[s>[s]]^{\alpha} P[s>[\theta]]^{\beta} P[z>[s]]^{\gamma} P[z>[\theta]]^{\delta} .
\end{aligned}
$$

Si se denota por $N$ el número de observaciones en la muestra, entonces $N=\alpha+\beta+\gamma+\delta$, y la expresión anterior queda: 


$$
\frac{N !}{\alpha ! \beta ! \gamma ! \cdot(N-\alpha-\beta-\gamma) !} P[s>[s]]^{\alpha} P[s>[\theta]]^{\beta} P[z>[s]]^{\gamma} P[z>[\theta]]^{\delta} .
$$

Tenemos así el número de permutaciones posibles entre los cuatro elementos $(s>[s], s>[\theta], z>[s]$ y $z>[\theta])$ para $N$ observaciones individuales, incluyendo la variación interna de cada uno de ellos.

Lo que resta, pues, es relacionar $P[s>[s]], P[s=[\theta]], P[z$ $[s]]$ y $P[z>[\theta]]$ con las proporciones individuales de realización de $/ \theta \mathrm{s} /$ según las pautas prototípicas de seseo $(\mathrm{S})$, ceceo $(\mathrm{C})$ y distinción (B); esto es, respectivamente, $P S, P C$ y $P B$, para poder utilizar esta distribución de probabilidad. Por el teorema de la probabilidad total, una probabilidad se puede separar como la suma de las probabilidades de partes disjuntas $\mathrm{y}$, por lo tanto, $P[s>[s]], P[s>[\theta]], P[z>[s]]$ y $P[z>[\theta]]$ se pueden reescribir como:

$$
\begin{aligned}
& P[s>[s]]=P[s>[s], s]+P[s>[s], C]+P[s>[s], B]= \\
& =P[s>[s] \mid S] \cdot P S+P[s>[s] \mid C] \cdot P C+P[s>[s] \mid B] \cdot P B .
\end{aligned}
$$

De ahí, recurriendo a los valores que ya se fijaron para cada una de las realizaciones en cada norma ideal, se obtiene la siguiente expresión:

$$
P[s>[s]]=1 \cdot P S+0 \cdot P C+1 \cdot P B=P S+P B,
$$

De la misma forma se llega a:

$$
\begin{gathered}
P[s>[\theta]]=P C \\
P[z>[s]]=P S \\
P[z>[\theta]]=P C+P B .
\end{gathered}
$$

Por lo tanto, el modelo probabilístico que relaciona $P S, P C$ y $P B$ con los datos muestrales es, sustituyendo en la última expresión conseguida para la distribución de probabilidad:

$$
\begin{aligned}
P[N s a s=\alpha, N s a z & =\beta, N z a s=\gamma, N z a z=\delta]= \\
& =\frac{N !}{\alpha ! \beta ! \gamma !(N-\alpha-\beta-\gamma) !}(P B+P S)^{\alpha} P C^{\beta} P S^{\gamma}(P B+P C)^{\delta},
\end{aligned}
$$

que da la probabilidad de cierto número de ocurrencias de cada tipo, suponiendo conocidos $P S, P C$ y $P B$.

Pero lo que se buscaba era estimar $P C, P S$ y $P B$, esto se logrará mediante el método denominado Estimador de Máxima Verosimilitud. Lo que hace este estimador es encontrar aquella configuración de los valores de los parámetros de la distribución ( $P S, P C$ y $P B$, en este caso) que asigna máxima probabilidad a la configuración do resultados (Nsas, Nsaz, Nzas, Nzaz) obtenidos on la 
muestra. Cuando hay un numero bastante alto de elementos observados para cada individuo, como en nuestro caso, este estimador es muy fiable.

Visualizando gráficamente el caso de una variable aleatoria que depende de un único parámetro que se denotará por par, $X_{\text {par, }}$ el estimador de máxima verosimilitud del parámetro par de la variable aleatoria $X$, fijado el resultado de una muestra, $\vec{x}$, viene dado por el punto del campo de los posibles valores del parámetro (que suponemos el intervalo $[a, b]$ en el gráfico) que tiene mayor verosimilitud, es decir, por el punto del campo de definición que asigna mayor probabilidad a la ocurrencia obtenida.

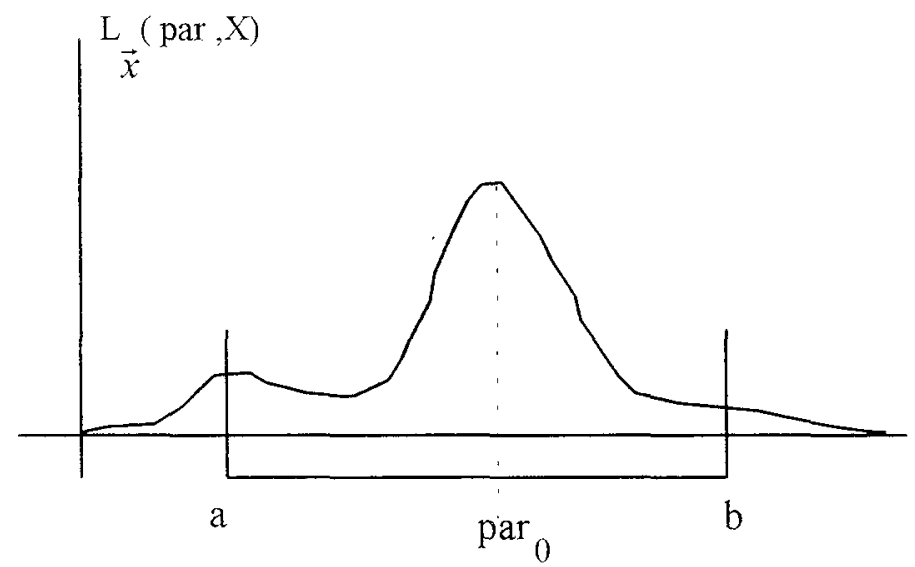

Gráfico 3: Estimador de máxima verosimilitud del parámetro par de la variable cuya función de verosimilitud para la muestra $\vec{x}$ es el valor de par $_{0}$

Supóngase que en una muestra se han obtenido los valores Nsas $=$ $\alpha, N s a z=\beta, N z a s=\gamma$ y $N z a z=\delta$, y se supone $P S, P C$ y $P B$ variables, con lo que queda la llamada función de verosimilitud, que es la función de probabilidad en función de los parámetros:

$$
L[P S, P C, P B]=\frac{N !}{\alpha ! \cdot \beta ! \cdot \gamma ! \cdot \delta !}(P S+P B)^{\alpha} P C^{\beta} P S^{\gamma}(P C+P B)^{\gamma} .
$$

Para simplificar los cálculos, se aplica el logaritmo natural a esta función; esto se puede llevar a cabo porque la función logaritmo es creciente, $y$, por lo tanto, $L[P S, P C, P B]$ y $\log (L[P S, P C, P B])$ tienen $\operatorname{los}$ mismos maximos y mínimos. La expresión obtenida es la siguiente:

$$
\log L[P S, P C, P B]=\log \left(\frac{N !}{\alpha ! \beta ! \gamma ! \delta !}(P S+P B)^{\alpha} P C^{\beta} P S^{\gamma}(P C+P B)^{\gamma}\right)
$$


Aplicando las propiedades del logaritmo que convierte el logaritmo del producto en suma de $\log$ aritmos $(\log (a \cdot b)=\log (a)+\log (b))$, y el logaritmo de una potencia es el exponente por el logaritmo de la base $\left(\log \left(a^{b}\right)=b \cdot \log (a)\right)$, se llega a la expresión:

$$
\begin{aligned}
\log L[P S, P C, P B]= & c t e+\alpha \log (P B+P S)+\beta \log P C+ \\
& +\gamma \log P S+\delta \log (P B+P C) .
\end{aligned}
$$

Puesto que, fijados los valores de $\alpha, \beta, \gamma$ y $\delta$, el valor de $N ! /(\alpha ! \cdot \beta ! \cdot \gamma ! \cdot \delta !)$ permanece constante y no tiene relevancia a la hora de operar, se ha sustituido esta expresión por la constante cte.

Dado que $P S, P C$ y $P B$ forman las proporciones de realización, se debe tener que $P S+P C+P B=1$, con lo que, utilizando esta igualdad, se obtiene que $P B=1-P C-P S$, lo cual, sustituido en la expresión anterior, equivale a:

$\log L[P S, P C]=c t e+\alpha \log (1-P C)+\beta \quad \log P C+\gamma \log P S+\delta \quad \log (1-P S)$.

Si se llevan a cabo las derivadas parciales con respecto a las variables $P S$ y $P C$ para poder encontrar los valores que maximizan la verosimilitud, se obtienen las expresiones siguientes:

$$
\begin{aligned}
& \frac{\partial}{\partial P S} \log L=0+0+0+\frac{\gamma}{P S}-\frac{\delta}{1-P S}=\frac{\gamma}{P S}-\frac{\delta}{1-P S} \\
& \frac{\partial}{\partial P C} \log L=0-\frac{\alpha}{1-P C}+\frac{\beta}{P C}+0+0=\frac{\beta}{P C}-\frac{\alpha}{1-P C}
\end{aligned}
$$

e igualando a cero, quedan las ecuaciones:

$$
\begin{aligned}
& \frac{\gamma}{P S}-\frac{\delta}{1-P S}=0 \\
& \frac{\beta}{P C}-\frac{\alpha}{1-P C}=0
\end{aligned}
$$

de donde, despejando:

$$
\begin{aligned}
& \gamma \quad(1-P S)=\delta \quad P S \Rightarrow \gamma=(\delta+\gamma \quad) P S \Rightarrow P S=\frac{\gamma}{\delta+\gamma} \\
& \beta \quad(1-P C)=\alpha \quad P C \Rightarrow \beta=(\alpha+\beta) P C \Rightarrow P C=\frac{\beta}{\alpha+\beta}
\end{aligned}
$$

con lo que se obtienen las estimaciones:

$$
P C=\frac{N s a z}{N s a s+N s a z} \quad P S=\frac{N z a s}{N z a s+N z a z} .
$$


Estas expresiones, a pesar de la aparente complejidad de su elaboración, tienen una interpretación bastante simple, y es que la probabilidad de ceceo es la proporción de $|\mathrm{s}|$ etimológicas pronunciadas $[\theta]$ ( $N s a z$ ) respecto del número total de $|s|(N s a z+N s a s)$, y de la misma forma, la probabilidad de seseo es la proporción de $|\theta|$ etimológicas realizadas [s] (Nzas) respecto del número total de $|\theta|$. La probabilidad de distinción queda determinada por estas dos proporciones, ya que se debe tener que $P S+P C+P B=1$ y, por lo tanto, se obtiene que $P B=1-P C-P S$. Hay que hacer notar que las proporciones que se han aplicado hasta el presente para calcular estas estimaciones han sido proporciones de realizaciones respecto al total de los datos, cuyo resultado es bastante distinto del hallado mediante las expresiones obtenidas, como se comprobará más adelante en este documento.

No es un error obtener que las estimaciones de $P S$ y $P C$ dependan únicamente de las realizaciones de $|\theta|$ y $|\mathbf{s}|$, respectivamente, ya que la estimación del modelo está formada por tres valores, $P S, P C$ y $P B$, y son los tres valores conjuntos los que determinan el comportamiento lingüístico del sujeto, con lo que no se pierde información, ya que el modelo estudiado es un modelo de Seseo, Ceceo y Distinción (aunque la distinción no se indicara explícitamente en el título, en el desarrollo se estableció como una probabilidad separada de $P S$ y $P($ ). Si se omitiera alguna de estas cantidades y se limitara el estudio a una sola de las estimaciones, el análisis sería parcial y en ninguna manera completo. Para el caso en que se quiera estudiar la Distinción frente a la Confusión de $s / z$, en la sección 4.2 de este trabajo se puede encontrar un desarrollo similar al de Seseo-Ceceo(-Distinción).

Hay que tener en cuenta que los valores estimados deben estar en el intervalo $[0,1]$, ya que son probabilidades, y en caso de no estar en este intervalo, se ha de tomar el valor en los extremos de $/ 0.1]$ que más verosimilitud tenga. Esta no es una aproximación sin sentido o subjetiva, sino que tiene una explicación clara e intuitiva. Si la estimación obtenida mediante el proceso anterior queda fuera del intervalo $[0,1]$, quiere decir que dentro de este intervalo no existe un máximo local como se mostró en el gráfico 3 , pero csto no significa que dentro del intervalo no haya un valor que tome el máximo valor en el intervalo, sino únicamente que no es un máximo local de la función. Entonces estaríamos en el caso del gráfico 4 : 


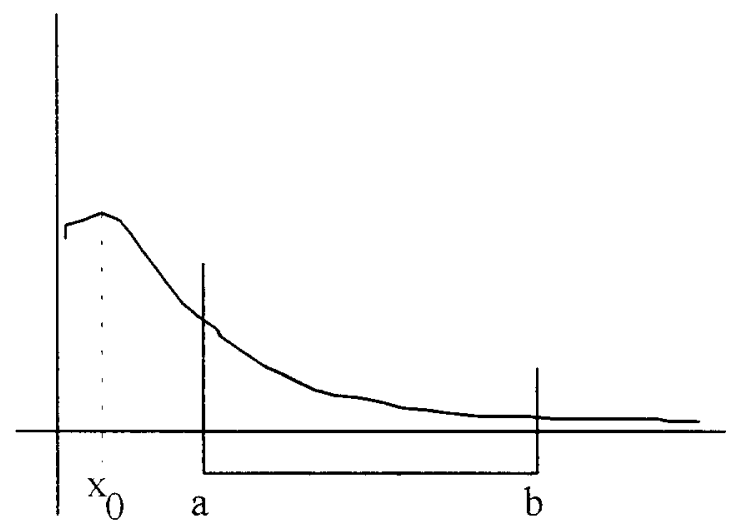

Gráfico 4: El máximo de la función se encuentra en $x_{0}$, pero este valor está fuera del intervalo de definición [a,b]; el valor máximo de la función en este intervalo se toma en $x=a$.

Por sus definiciones, $P C$ y $P S$ siempre están en el intervalo $[0,1]$ (la suma de dos términos positivos siempre es mayor que una de las partes, y por lo tanto, los numeradores siempre van a ser más pequeños que los denominadores, de modo que $P S$ y $P C$ serán menores que uno; además, tanto los numeradores como los denominadores van a ser no negativos, y de esta manera $P S$ y $P C$ van a ser mayores que cero). Sólo falta por comprobar $P B$, que tiene la expresión:

$$
\begin{aligned}
P B=1-P C & -P S= \\
& =1-\frac{N s a z}{N s a s+N s a z}-\frac{N z a s}{N z a s+N z a z},
\end{aligned}
$$

y para que $P B$ esté en el intervalo $[0,1]$, debe estarlo:

$$
\begin{aligned}
& \frac{N s a z}{N s a s+N s a z}-\frac{N z a s}{N z a s+N z a z}= \\
& =\frac{N s a z \cdot N z a z+N z a s \cdot N s a s+2 \cdot N s a z \cdot N z a s}{(N s a s+N s a z)(N z a s+N z a z)} .
\end{aligned}
$$

Para ello se deben dar las condiciones:

(1) $N_{s a z} \cdot N z a z+N z a s \cdot N s a s+2 \cdot N s a z \cdot N z a s \geq 0$, para que $P B \leq 1$.

(2) $N s a z \cdot N z a s \leq N s a s \cdot N z a z$, para que $P B \geq 0$.

La condición (1) siempre se verificará, por lo que se puede eliminar, y la condición (2), caso de no verificarse, implicaría que $P B<0$, con lo que se haría $P B=0$. Se debe ajustar el nuevo modelo de probabilidad de la misma forma, sólo que ahora $P S+P C=1$, ya que $P B=0$. 
Sustituyendo en la función de verosimilitud $P B=0$, y utilizando que $P C=1-P S$, la expresión de la función de $\log L$ quedaría:

$$
\log L=c t e+(\alpha+\gamma) \log P S+(\beta+\delta) \log (1-P S),
$$

y encontrando el estimador de máxima verosimilitud para $P S$ :

$$
\frac{\partial}{\partial P S} \log L=0+\frac{\alpha+\gamma}{P S}-\frac{\beta+\delta}{1-P S},
$$

que igualando a cero:

$$
\frac{\alpha+\gamma}{P S}=\frac{\beta+\delta}{1-P S},
$$

y despejando y sustituyendo en $P S+P C=1$, queda:

$$
\begin{aligned}
& P S=\frac{N s a s+N z a s}{N s a s+N s a z+N z a s+N z a z} \\
& P C=\frac{N z a z+N s a z}{N s a s+N s a z+N z a s+N z a z},
\end{aligned}
$$

con lo que ya se tendrían los estimadores cuando se tuviera que hacer $P B=0$.

Resumiendo los resultados en un único esquema, quedaría:

(1) Si se tiene Nsas. Nzaz $\geq N z a s \cdot N s a z$, las estimaciones tienen la forma:

$$
\begin{gathered}
P S=\frac{N z a s}{N z a z+N z a s} \quad P C=\frac{N s a z}{N s a z+N s a s} \\
P B=I-P S-P C
\end{gathered}
$$

(2) Si por el contrario Nsas. Nzaz $\leq$ Nzas. Nsaz, toman la forma:

$$
P S=\frac{N s a s+N z a s}{N s a s+\begin{array}{l}
N s a z+N z a s+N z a z \\
N s a s+N z a s
\end{array}}
$$

Por ejemplo, si en una muestra se obtienen los resultados: Nsas = $112, N s a z=0, N z a s=0$ y $N z a z=135$, utilizando estos valores se elige entre las dos opciones comparando la expresión Nsas $\cdot \mathrm{Nzaz}=112 \cdot 135=15120$ con la expresión $N s a z$. Nzas $=0 \cdot 0=0, y$ puesto que Nsas. Nzaz $\geq N z a s \cdot N s a z$, se llevan a cabo los cálculos según las expresiones (1): 


$$
\begin{aligned}
& P S=\frac{N z a s}{N z a z+N z a s}=\frac{0}{135+0}=\frac{0}{135}=0 \\
& P C=\frac{N s a z}{N s a z+N s a s}=\frac{0}{112+0}=\frac{0}{112}=0 \\
& P B=1-P S-P C=1-0-0=1 .
\end{aligned}
$$

Los resultados obtenidos son los esperados, ya que si una persona no confunde, se le asigna una probabilidad de distinción PB máxima, y probabilidades de seseo y ceceo mínimas.

$\mathrm{Si}$ en la muestra se han obtenido los valores $N s a s=18, N s a z=50, N z a s$ $=0$, y $N z a z=46$, utilizando estos valores se elige entre las dos opciones comparando el resultado de la expresión $N s a s \cdot N z a z=18 \cdot 46=828$ con el de la expresión $N s a z \cdot N z a s=0 \cdot 50=0$, y puesto que $N_{s a s} \cdot N z a z \geq N z a s \cdot N s a z$, se llevan a cabo los cálculos mediante las ecuaciones (1):

$$
\begin{aligned}
& P S=\frac{N z a s}{N z a z+N z a s}=\frac{0}{46+0}=\frac{0}{46}=0 \\
& P C=\frac{N s a z}{N s a z+N s a s}=\frac{50}{50+18}=\frac{50}{68} \approx 0.735 \\
& P B=1-P S-P C \approx 1-0-0,735 \approx 0.25
\end{aligned}
$$

El simbolo $\approx$ significa aproximadamente, ya que los valores decimales expuestos son aproximados. Se puede ver en este caso cómo la probabilidad de ceceo es alta, y la de seseo nula, por lo que la probabilidad de distinción es algo baja. Aquí está la clave de por qué se han de considerar las tres probabilidades al mismo tiempo, y no por separado: las tres probabilidades interactúan en el modelo probabilístico, y por lo tanto a la hora de ser calculadas, y el modelo sólo tiene sentido si se calculan las tres a la vez; en otro caso, se lleva a cabo un estudio sesgado y probablemente con resultados erróneos. De esta forma, aunque en el cálculo de las estimaciones de PC y PS únicamente actúen las $|\mathrm{s}|$ y las $|\theta|$ etimológicas, respectivamente, el individuo queda totalmente caracterizado desde el punto de vista de la pronunciación con el conjunto de las tres probabilidades PS, PC y $P B$, ya que así quedan recogidas todas sus facetas.

Como último ejemplo, para el caso en el que $N s a s=9, N s a z=125$, Nzas $=2$ y $N z a z=27$, se elige entre las dos opciones comparando la expresión $N$ sas $N_{z a z}=9 \cdot 27 \cdots 243$ con la expresión $N s a z \cdot N z a S=125 \cdot 2=250$, y puesto 
que Nsas-Nzaz $\leq N z a s \cdot N s a z$, se llevan a cabo los cálculos mediante las ecuaciones (2):

$$
\begin{aligned}
& P S=\frac{N s a s+N z a s}{N s a s+N s a z+N z a s+N z a z}=\frac{9+2}{9+125+2+27}=\frac{11}{163} \approx 0,067 \\
& P C=\frac{N s a z+N z a z}{N s a s+N s a z+N z a s+N z a z}=\frac{125+27}{9+125+2+27}=\frac{152}{163} \approx 0,9325 \\
& P B=0
\end{aligned}
$$

Como era de esperar, debido a las puntuaciones obtenidas, el sujeto en cuestión no distingue en absoluto $(\mathrm{PB}=0$ ), cecea de una manera bastante evidente $(\mathrm{PC}=0.923)$ y sesea en una pequeña proporción $(\mathrm{PS}=0.0769)$.

En la sección 5 de este trabajo se presentan los resultados obtenidos a partir del estudio de una red social del barrio de Capuchinos de Málaga.

\subsection{Modelo de Distinción-Confusión.}

En el modelo anterior de Seseo-Ceceo(-Distinción) se ha estudiado la interrelación de tres normas de comportamiento lingüístico, correspondientes a los conceptos de Seseo, Ceceo y Distinción. Si se pretende estudiar el grado de Confusión y Distinción de un hablante, hay que construir un nuevo modelo, en el que la Confusión y la Distinción vengan identificadas por nuevos patrones de comportamiento. La opción más frecuente consiste en sumar las realizaciones $s>[\theta]$ y $z>[s]$, y considerar su proporción con respecto al total como la frecuencia o índice de confusión; consecuentemente, uno menos esa cantidad constituye la proporción de distinción o acierto. Se intentará mejorar esta estimación siguiendo un método similar al del apartado anterior. A esto se añade otro problema ya tratado por Ávila (1994) y comentado arriba (párr. 2.3). En situaciones claramente bipolarizadas, como la del barrio de Capuchinos con respecto a la variación de $/ \theta \mathrm{s} /$, con realizaciones de $/ \theta \mathrm{s} />[\mathrm{s}]$ predominantemente femeninas y de $/ \theta \mathrm{S} />[\theta]$ predominantemente masculinas (Villena y Requena 1994), la suma antedicha, al partir de las frecuencias brutas en las que el número de $|s|$ etimológicas es mayor que el número de $|z|$ etimologicas, produce sesgos importantes, puesto que los individuos que tienden a la pauta $S(s>[s]$ y $z>[s])$ resultan más conservadores (menos confundidores) que los que tienden a la pauta $\mathrm{C}(\mathrm{z}>[\theta]$ y $s>[\theta])$ : 


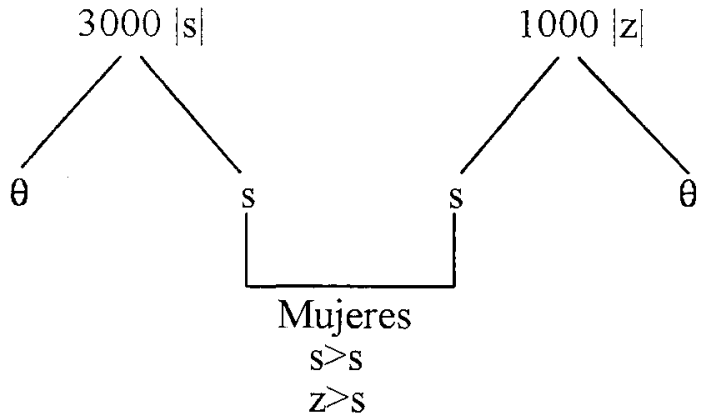

Conviene, en primer lugar, especificar qué se entiende por confusión y qué se entiende por distinción:

- Un distinguidor prototípico se supone un individuo que únicamente lleva a cabo las realizaciones $s>[s]$ y $z>[\theta]$, lo cual se podría expresar mediante probabilidades condicionadas de la realización frente a la etimología como:

\begin{tabular}{c|c|c|}
$\mathrm{P}[$ Realización | etimología] & \multicolumn{2}{|c|}{ Realización } \\
\hline Etimología & {$[\mathrm{s}]$} & {$[\theta]$} \\
\hline $\mathrm{s}$ & 1 & 0 \\
\hline $\mathrm{z}$ & 0 & 1 \\
\hline
\end{tabular}

- Un confundidor prototípico llevaría a cabo ambas realizaciones posibles para cada etimología, tal como refleja la siguiente tabla de probabilidades:

\begin{tabular}{c|c|c|}
$\mathrm{P}[$ Realización | etimología] & \multicolumn{2}{|l|}{ Realización } \\
\hline Etimología & {$[\mathrm{s}]$} & {$[\theta]$} \\
\hline $\mathrm{s}$ & 0.5 & 0.5 \\
\hline $\mathrm{z}$ & 0.5 & 0.5 \\
\hline
\end{tabular}

De nuevo, como en el caso del modelo de Seseo-Ceceo(-Distinción), tenemos que hay realizaciones coincidentes con las etimológicas que pueden no deberse al modelo de distinción, sino que pueden provenir de una realización canónica o etimológica casual. Ésta es la razón por la que no se pueden considerar exactas las estimaciones de las proporciones de confusión y distinción expresadas al principio de este apartado.

La elaboración del modelo probabilístico sigue un procedimiento similar al del caso anterior.

Se supone que el individuo realiza $/ \theta \mathrm{S} /$ según los modelos de distinción y confusión, unas veces a través de uno y otras veces a través del otro. 
Consideraremos como PD la proporción de veces que el sujeto realiza $/ \theta$ s/ según el modelo de distinción, al cual se denotará por $\mathrm{D}$, y como PK la proporción de veces que realiza $/ \theta$ / según el modelo de confusión, al que se denotará por K. El comportamiento del sujeto estudiado se puede ver esquematizado en el siguiente gráfico:
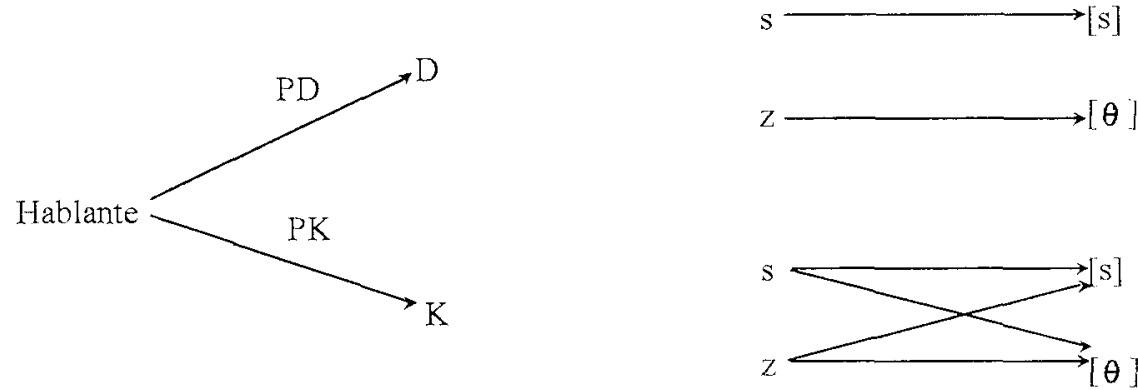

Gráfico 5: Modelo general de comportamiento según el modelo de

Distinción-Confusión

En el desarrollo del modelo anterior de Ceceo-Seseo(-Distinción), se construyó la distribución de probabilidad de las realizaciones $N s a s=\alpha, N s a z$ $=\beta, N z a s=\gamma$ y $N z a z=\delta$, supuestas conocidas las probabilidades $P[s>[s]]$, $P[s>[\theta]], P[z>[s]]$ y $P[z>[\theta]]$; en esta sección se empezará directamente a partir de esta distribución ya construida (para todo ello, véase párr. 4.1):

$$
\begin{aligned}
P[N \text { Nas }=\alpha, N s a z=\beta, N z a s=\gamma, N z a z=\delta]= \\
\quad=\frac{(\alpha+\beta+\gamma+\delta) !}{\alpha ! \beta ! \gamma ! \delta !} P[s>[s]]^{\alpha} P[s>[\theta]]^{\beta} P[z>[s]]^{\gamma} P[z>[\theta]]^{\delta} .
\end{aligned}
$$

Como en el caso anterior, se establecen a continuación las relaciones entre $P[s>[s]], P[z>[s]], P[z>[s]]$ y $P[z>[\theta]]$ y las probabilidades $P D y$ PK. Desarrollando de la misma manera que en el modelo de ceceo-seseodistinción, se obtiene:

$$
\begin{gathered}
P[s>[s]]=P[s>[s], D]+P[s>[s], K]= \\
=P[s>[s] \mid D] \cdot P D+P[s>[s] \mid K] \cdot P K= \\
=1 \cdot P D+0.5 \cdot P K
\end{gathered}
$$

y de la misma forma para los demás, se llega a: 


$$
\begin{gathered}
P[s>[s]]=P D+0.5 \cdot P K \\
P[s>[\theta]]=0.5 \cdot P K \\
P[z>[s]]=0.5 \cdot P K \\
P[z>[\theta]]=P D+0.5 \cdot P K,
\end{gathered}
$$

de donde, sustituyendo en la expresión de la multinomial, se obtiene:

$$
\begin{aligned}
P[N \text { sas }=\alpha, & \text { Nsaz }=\beta, N z a s=\gamma, N z a z=\delta]= \\
= & \frac{(\alpha+\beta+\gamma+\delta) !}{\alpha ! \beta ! \gamma ! \delta !}(P D+0.5 \cdot P K)^{\alpha+\delta}(0.5 \cdot P K)^{\beta+\gamma} .
\end{aligned}
$$

De esta manera, se tiene ya la distribución de probabilidad de las ocurrencias en función de las proporciones PD y PK. A continuación, se encontrará la expresión del estimador de máxima verosimilitud de los parámetros de esta distribución, PD y PK. Para ello, primero se hacen logaritmos de la función de verosimilitud:

$$
L[P D, P K]=\frac{(\alpha+\beta+\gamma+\delta) !}{\alpha ! \beta ! \gamma ! \delta !}(P D+0.5 \cdot P K)^{\alpha+\delta}(0.5 \cdot P K)^{\beta+\gamma},
$$

con lo que queda:

$$
\log L[P D, P K]=c t e+(\alpha+\delta) \log (P D+0.5 \cdot P K)+(\beta+\gamma) \log (0.5 \cdot P K) .
$$

Si se utiliza la relación $P D+P K=1$, de donde se obtiene la igualdad $P D=1-P K$, queda:

$$
\log L[P K]=c t e+(\alpha+\delta) \log (1-0.5 \cdot P K)+(\beta+\gamma) \log (0.5 \cdot P K) .
$$

Derivando con respecto a PK se obtiene:

$$
\frac{\partial}{\partial P K} \log L[P K]=0-0.5 \frac{\alpha+\delta}{l-0.5 \cdot P K}+0.5 \frac{\beta+\gamma}{0.5 \cdot P K},
$$

de donde, igualando a cero y operando, resulta la siguiente ecuación:

$$
\frac{\alpha+\delta}{I-0.5 \cdot P K}=\frac{\beta+\gamma}{0.5 \cdot P K} \text {. }
$$

Despejando se llega a la expresión de PK:

$$
P K=2 \cdot \frac{N s a z+N z a s}{N s a s+N s a z+N z a s+N z a z}
$$

y la expresión de PD es su complementario. Pero hay que tener en cuenta que el valor de $P K$ ha de estar en el intervalo $[0,1]$ (y al ser $P D=1-P K$, también 
lo estará), y se puede comprobar, tras cálculos algebraicos, que esto se cumplirá si se dan las dos siguientes inecuaciones:

(1) $N s a z+N z a s \geq 0$ para que $P K \geq 0$.

(2) Nsaz+Nzas $\leq N s a s+N z a z$ para que $P K \leq 1$.

De nuevo la condición (1) es evidente, por lo que se puede ignorar, mientras que si la condición (2) no se verifica, tenemos que $P K>1$, por lo que forzaríamos a $P K$ a tomar el máximo valor del intervalo, es decir, $P K=I$. Por lo tanto, el cálculo del estimador de máxima verosimilitud para los parámetros $\mathrm{PK}$ y $\mathrm{PD}$ se resume en el siguiente esquema:

- Si Nsaz+Nzas $\leq N s a s+N z a z$ :

- Si Nsaz+Nzas> Nsas+Nzaz:

$$
\begin{gathered}
P K=2 \cdot \frac{N s a z+N z a s}{N s a s+N s a z+N z a s+N z a z} \\
P D=\frac{N s a s+N z a z-N s a z-N z a s}{N s a s+N s a z+N z a s+N z a z}
\end{gathered}
$$

$$
P K=1, \quad P D=0
$$

Este esquema se utilizaría en la práctica como en la sección 4.1.

La interpretación de estas expresiones no es tan clara como en el modelo de Seseo-Ceceo(-Distinción). Se puede indicar que la proporción de confusión es el doble de la proporción de realizaciones en las que la etimología no coincide con la realización ( $N s a z+N z a s$ ), frente al total de los datos ( $N s a s$ $N s a z+N z a s+N z a z$ ), salvo que sobrepase a uno, en cuyo caso se restringe su valor al máximo posible (uno), mientras que el procedimiento generalmente usado de recuento le asignaba la proporción:

$$
\frac{N s a z+N z a s}{N s a s+N s a z+N z a s+N z a z},
$$

que evidentemente es menor que la probabilidad obtenida con el modelo aquí expuesto.

En lo que sigue, se hace una exposición de los resultados obtenidos para los datos de la red de Capuchinos tratada en la investigación de Ávila (1994).

\section{APLICACIÓN}

La red de Capuchinos estudiada por Ávila (1994) presenta un total de 30 sujetos $(H=12, M=18)$, con diferencias de edad, sexo y posición social. E1 número total de ocurrencias de $/ \theta \mathrm{s} /$ analizadas fue de 4777 .

A continuación se da una tabla donde se indican las variables: Sujeto, Nsas, Nsaz, Nzas y Nzaz, y en la que Sujeto es un número asociado a cada individuo para su identificación; las restantes son las ya especificadas: 


$\begin{array}{ccccc}\text { Sujeto } & \text { Nsas } & \text { Nsaz } & \text { Nzas } & \text { Nzaz } \\ 1 & 112 & 0 & 0 & 135 \\ 2 & 58 & 0 & 0 & 37 \\ 3 & 18 & 50 & 0 & 46 \\ 4 & 9 & 105 & 0 & 27 \\ 5 & 9 & 115 & 0 & 55 \\ 6 & 5 & 79 & 0 & 80 \\ 7 & 36 & 77 & 2 & 110 \\ 8 & 92 & 24 & 4 & 205 \\ 9 & 113 & 0 & 5 & 134 \\ 10 & 67 & 0 & 4 & 50 \\ 11 & 95 & 1 & 7 & 77 \\ 12 & 39 & 14 & 5 & 52 \\ 13 & 21 & 37 & 9 & 73 \\ 14 & 15 & 26 & 7 & 50 \\ 15 & 86 & 6 & 7 & 45 \\ 16 & 34 & 77 & 7 & 38 \\ 17 & 35 & 95 & 6 & 27 \\ 18 & 70 & 0 & 20 & 69 \\ 19 & 57 & 0 & 6 & 15 \\ 20 & 56 & 4 & 16 & 23 \\ 21 & 91 & 11 & 18 & 23 \\ 22 & 52 & 2 & 34 & 11 \\ 23 & 76 & 0 & 45 & 12 \\ 24 & 92 & 0 & 31 & 4 \\ 25 & 90 & 1 & 91 & 11 \\ 26 & 113 & 0 & 36 & 4 \\ 27 & 115 & 2 & 43 & 3 \\ 28 & 125 & 2 & 31 & 2 \\ 29 & 62 & 3 & 62 & 2 \\ 30 & 132 & 0 & 85 & 2\end{array}$

La elaboración de los datos se ha llevado a cabo con el paquete estadístico SPSS; a continuación se expone el código utilizado para calcular PB, PC, PS, PK y PD:

$$
\begin{array}{ll}
\text { COMPUTE } & \text { NN =Nsas } * \text { Nzaz }- \text { Nsaz } * \text { Nzas } \\
\text { lF }(N N>=0) & P C=N s a z /(\text { Nsaz }+ \text { Nsas }) \\
\text { IF }(N N>=0) & P S=\text { Nzas } /(\text { Nzas }+ \text { Nzaz })
\end{array}
$$




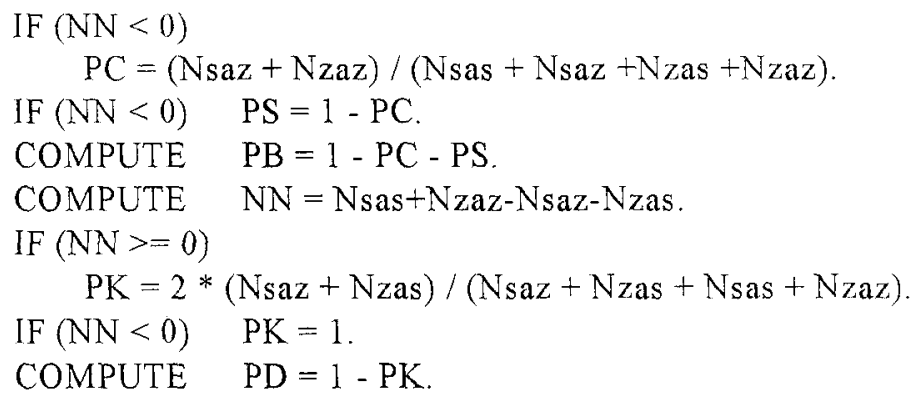

La variable $\mathrm{NN}$ es únicamente una variable auxiliar que permite saber qué expresión del estimador de máxima verosimilitud hay que utilizar.

Los resultados de la aplicación de los estimadores desarrollados en este trabajo se pueden ver en la siguiente tabla. En ella se exponen con fines comparativos dichos resultados (PS, $\mathrm{PC}, \mathrm{PB}, \mathrm{PK}, \mathrm{PD}$ ), junto con las proporciones ( $\mathrm{PSr}, \mathrm{PCr}, \mathrm{PBr}, \mathrm{PDr}, \mathrm{PKr}$ ), correspondientes al método de recuento de errores y aciertos utilizado hasta ahora $^{33}$. Las expresiones de esos últimos estimadores son:

- $P_{c r}=N_{s a z} / N, P_{s r}=N z a s / N$ y $P B r=1-P_{c r}-P S r$ para el modelo de seseo-ceceo-distinción.

$-P k r=(N s a z+N z a s) /(N s a s+N s a z+N z a s+N z a z)$ y $P D r=1-P K r$ para el modelo de confusión distinción.

31 En otros trabajos se pueden encontrar estimaciones que consideran por separado las realizaciones de la $|s|$ etimológica y de la $|z|$ etimológica, sin construir necesariamente un modelo de Ceceo-Seseo(-Distinción ), sino dos modelos que estudian el mantenimiento o el trueque de la |s y y la $|z|$ etimológicas, cada una por separado. Este no es el tipo de estudio que se ha planteado aquí. 


\begin{tabular}{|c|c|c|c|c|c|c|c|c|c|c|c|c|c|c|}
\hline Sujcto & Nsas & Nsaz & Nzas & Nzaz & PS & $\mathrm{PC}$ & PB & $\mathrm{PSr}$ & $\mathrm{PCr}$ & $\mathrm{PBr}$ & $\mathrm{PK}$ & PD & $\mathrm{PKr}$ & $\mathrm{PDr}$ \\
\hline$I$ & 112 & 0 & 0 & 135 & 0,00 & 0,00 & 1,00 & 0,00 & 0,00 & 1,00 & 0,00 & 1,00 & 0,00 & 1,00 \\
\hline 2 & 58 & 0 & 0 & 37 & 0,00 & 0,00 & 1,00 & 0,00 & 0,00 & 1,00 & 0,00 & 1,00 & 0,00 & 1,00 \\
\hline 3 & 18 & 50 & 0 & 46 & 0,00 & 0,74 & 0,26 & 0,00 & 0,44 & 0,56 & 0,88 & 0,12 & 0,44 & 0,56 \\
\hline 4 & 9 & 105 & 0 & 27 & 0,00 & 0,92 & 0,08 & 0,00 & 0,74 & 0,26 & 1,00 & 0,00 & 0,74 & 0.26 \\
\hline 5 & 9 & 115 & 0 & 55 & 0,00 & 0,93 & 0.07 & 0,00 & 0.64 & 0,36 & 1,00 & 0,00 & 0,64 & 0,36 \\
\hline 6 & 5 & 79 & 0 & 80 & 0,00 & 0,94 & 0,06 & 0,00 & 0,48 & 0,52 & 0,96 & 0,04 & 0,48 & 0,52 \\
\hline 7 & 36 & 77 & 2 & 110 & 0,02 & 0,68 & 0,30 & 0,01 & 0,34 & 0,65 & 0,70 & 0,30 & 0,35 & 0.65 \\
\hline 8 & 92 & 24 & 4 & 205 & 0,02 & 0,21 & 0,77 & 0,01 & 0,07 & 0,91 & 0,17 & 0,83 & 0,09 & 0,91 \\
\hline 9 & 113 & 0 & 5 & 134 & 0,04 & 0,00 & 0,96 & 0,02 & 0,00 & 0,98 & 0,04 & 0,96 & 0,02 & 0,98 \\
\hline 10 & 67 & 0 & 4 & 50 & 0,07 & 0,00 & 0,93 & 0,03 & 0,00 & 0,97 & 0,07 & 0,93 & 0,03 & 0,97 \\
\hline 11 & 95 & 1 & 7 & 77 & 0,08 & 0,01 & 0,91 & 0,04 & 0,01 & 0,96 & 0,09 & 0,91 & 0,04 & 0.96 \\
\hline 12 & 39 & 14 & 5 & 52 & 0,09 & 0,26 & 0,65 & 0,05 & 0,13 & 0,83 & 0,35 & 0,65 & 0,17 & 0,83 \\
\hline 13 & 21 & 37 & 9 & 73 & 0,11 & 0,64 & 0,25 & 0,06 & 0,26 & 0,67 & 0,66 & 0,34 & 0,33 & 0,67 \\
\hline 14 & 15 & 26 & 7 & 50 & 0,12 & 0,63 & 0,24 & 0,07 & 0,27 & 0,66 & 0,67 & 0,33 & 0,34 & 0,66 \\
\hline 15 & 86 & 6 & 7 & 45 & 0,13 & 0,07 & 0,80 & 0,05 & 0,04 & 0,91 & 0,18 & 0,82 & 0,09 & 0,91 \\
\hline 16 & 34 & 77 & 7 & 38 & 0,16 & 0,69 & 0,15 & 0,04 & 0,49 & 0,46 & 1,00 & 0,00 & 0,54 & 0,46 \\
\hline 17 & 35 & 95 & 6 & 27 & 0,18 & 0,73 & 0,09 & 0,04 & 0,58 & 0,38 & 1,00 & 0,00 & 0,62 & 0,38 \\
\hline 18 & 70 & 0 & 20 & 69 & 0,22 & 0,00 & 0,78 & 0,13 & 0,00 & 0,87 & 0,25 & 0,75 & 0,13 & 0,87 \\
\hline 19 & 57 & 0 & 6 & 15 & 0,29 & 0,00 & 0,71 & 0,08 & 0,00 & 0,92 & 0,15 & 0,85 & 0,08 & 0,92 \\
\hline 20 & 56 & 4 & 16 & 23 & 0,41 & 0,07 & 0,52 & 0,16 & 0,04 & 0,80 & 0,40 & 0,60 & 0,20 & 0,80 \\
\hline 21 & 91 & 11 & 18 & 23 & 0,44 & 0,11 & 0,45 & 0,13 & 0,08 & 0,80 & 0,41 & 0,59 & 0,20 & 0,80 \\
\hline 22 & 52 & 2 & 34 & 11 & 0,76 & 0,04 & 0,21 & 0,34 & 0,02 & 0,64 & 0,73 & 0,27 & 0,36 & 0,64 \\
\hline 23 & 76 & 0 & 45 & 12 & 0,79 & 0,00 & 0,21 & 0,34 & 0,00 & 0,66 & 0,68 & 0,32 & 0,34 & 0,66 \\
\hline 24 & 92 & 0 & 31 & 4 & 0,89 & 0,00 & 0,11 & 0,24 & 0,00 & 0,76 & 0,49 & 0,51 & 0,24 & 0,76 \\
\hline 25 & 90 & 1 & 91 & 11 & 0,89 & 0,01 & 0,10 & 0,47 & 0,01 & 0,52 & 0,95 & 0,05 & 0,48 & 0.52 \\
\hline 26 & 113 & 0 & 36 & 4 & 0,90 & 0,00 & 0,10 & 0,24 & 0,00 & 0,76 & 0,47 & 0,53 & 0,24 & 0,76 \\
\hline 27 & 115 & 2 & 43 & 3 & 0,93 & 0,02 & 0,05 & 0,26 & 0,01 & 0,72 & 0,55 & 0,45 & 0,28 & 0,72 \\
\hline 28 & 125 & 2 & $3 !$ & 2 & 0,94 & 0,02 & 0,04 & 0,19 & 0,01 & 0,79 & 0,41 & 0.59 & 0,21 & 0.79 \\
\hline 29 & 62 & 3 & 62 & 2 & 0,96 & 0,04 & 0,00 & 0,48 & 0,02 & 0,50 & 1,00 & 0,00 & 0,50 & 0,50 \\
\hline 30 & 132 & 0 & 85 & 2 & 0,98 & 0,00 & 0,02 & 0,39 & 0,00 & 0,61 & 0,78 & 0,22 & 0,39 & 0,61 \\
\hline
\end{tabular}

Por ejemplo, el individuo 30 , tiene una destacada tendencia al seseo, ya que, salvo dos excepciones, no hace más realizaciones que $s>[s]$ y $z>[s]$. En el modelo basado en las proporciones, se le asigna una probabilidad de $P_{S r^{\circ}}=$ 0.39, mientras que el modelo aquí desarrollado le asigna una probabilidad $P S$ $=0.98$. Este comportamiento se puede observar en diversos individuos. La mejor forma de ver la diferencia entre las asignaciones que realiza cada modelo es un gráfico. En los que se exponen a continuación (Gráficos 6 y 7 ), se puede 
observar una nube de puntos para cada uno de los modelos PS-PC y PSr-PCr; para su correcta lectura hay que tener en cuenta que $P S+P C+P D=1$, por lo que cuanto más cerca se encuentre un individuo del punto $(0,0)$, es decir, del origen de coordenadas, en el extremo inferior izquierdo, menos sumará $\mathrm{PC}$ y $P S$, de donde, debido a la relación $P S+P C+P B=1, P B$ estará cercano a 1 , con lo que su proporción de acierto será mayor. Por lo tanto, cuanta mayor proximidad al extremo superior izquierdo, mayor probabilidad de ceceo; cuanto más cerca del extremo inferior derecha, mayor probabilidad de seseo, y cuanto más cerca del extremo inferior izquierdo, mayor tendencia a la pronunciación canónica.
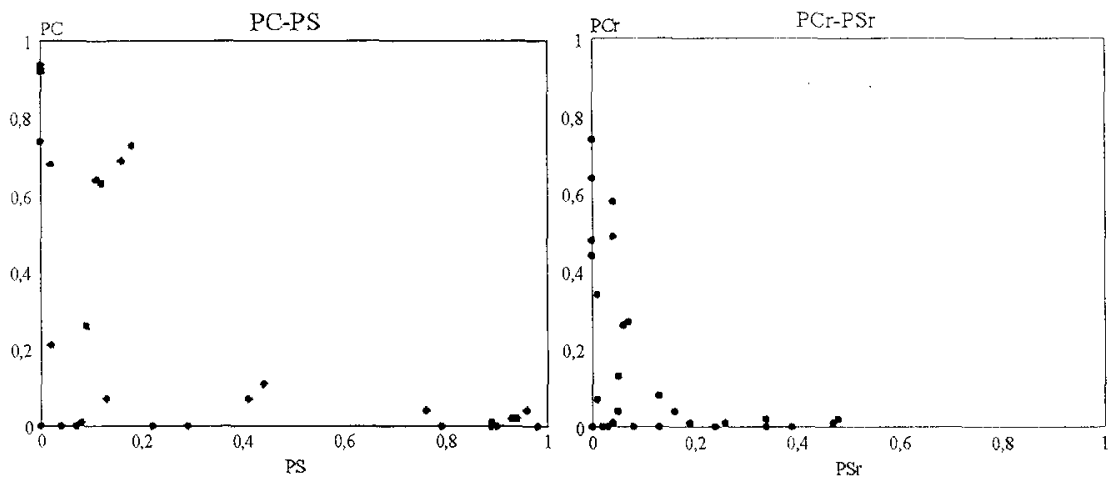

Gráfico 6: Comparación del comportamiento individual para la realización de $/ \theta^{\mathrm{S}} /$ en el modelo probabilístico (izq.) y de proporciones (dcha.) para el estudio del Seseo-Ceceo-Distinción.

Se puede observar cómo el modelo estudiado en este trabajo desarrolla más la nube de puntos hacia los extremos, mientras que el modelo $\mathrm{PSr}-\mathrm{PCr}$ comprime los datos, asignando más distinción a individuos que, como ya se ha indicado, confunden de una manera casi completa. Esto es debido al hecho ya indicado de que el modelo $\mathrm{PCr}-\mathrm{PSr}$ no tiene en cuenta las realizaciones canónicas debidas al azar.

Exactamente igual ocurre con el modelo PD-PK, frente a PDr-PKr, en el que los puntos tienden a concentrarse hacia la izquierda (menor probabilidad de confusión) en el modelo basado en proporciones, dando una imagen inexacta de la realidad. 

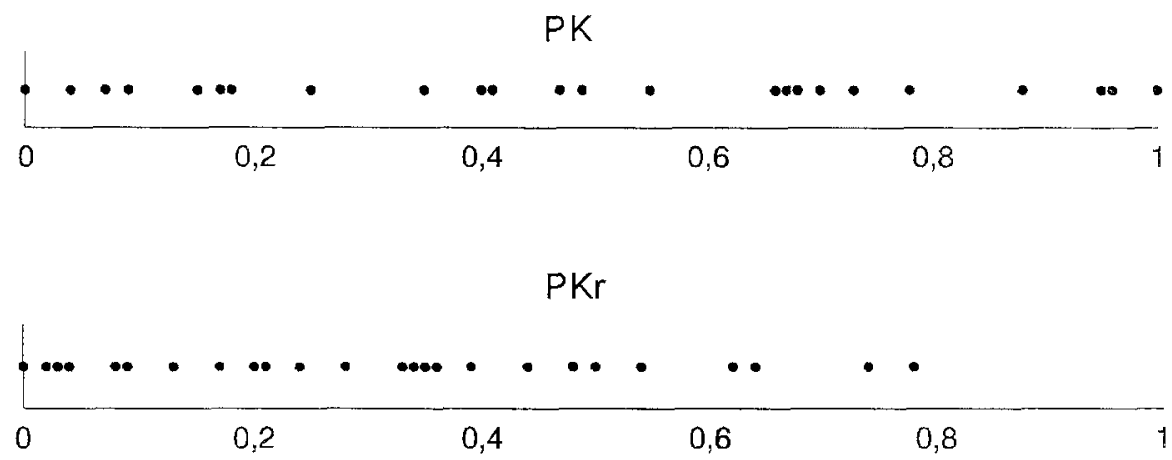

Gráfico 7: Comparación del comportamiento individual para la realización de $/ \theta^{s}$ en el modelo probabilístico (arriba) y de proporciones (abajo) para el estudio de la Confusión-Distinción.

\section{CONCLUSIONES}

Como se ha visto, los modelos probabilísticos de Seseo-Ceceo y Distinción-Confusión constituyen una base firme para la solución de los problemas que plantea el estudio de la realización de $/ \theta$ s/ en el español hablado. No obstante, hay que tener en cuenta que nuestras suposiciones iniciales son bastante fuertes, puesto que, como se ha visto en diversos estudios sobre otras variables similares, el entorno lingüístico puede condicionar una determinada realización. Sin embargo, parece evidente que sin las suposiciones adoptadas, sería prácticamente imposible llevar a cabo el tratamiento que se ha desarrollado en este trabajo, y el modelo se complicaría infinitamente, al tener que considerar todas las posibilidades del entorno. A ello se añade la dificultad específica de la variable analizada aquí.

La experiencia sobre trabajos cuantitativos para casos bivariantes, como la realización o pérdida de ciertas consonantes, es muy amplia y ha producido muy buenos resultados, pero no disponíamos de métodos similares a los utilizados en esos problemas que se pudieran aplicar al caso trivariante en cuestión.

Además, hay que añadir que, en este caso, las normas ideales de realización tienen realizaciones comunes, con lo que se mezclan en la muestra, de modo que hay que tener este hecho en cuenta para poder estimar correctamente el modelo. Esto implica una clara determinación probabilística del problema, que requiere un tratamiento probabilístico en lugar de estadístico descriptivo. Así mismo, esta variabilidad de las posibilidades imposibilita el uso de programas estadísticos como GoldVarb o SPSS de una forma directa, 
presentándose además el problema de que estos programas están diseñados para realizar la regresión logística (método que se utilizaría para realizar un ajuste de los resultados de las realizaciones frente a otras variables lingüísticas o sociales) únicamente para variables con dos valores, mientras que la variable de realización que aquí aparece puede tomar cuatro valores.

Los resultados encontrados parecen indicar que el modelo aquí desarrollado, así como las estimaciones que se obtienen de él, bajo las suposiciones hechas, es correcto y funciona bien, ajustándose a la realidad.

\section{REFERENCIAS}

- ALVAR, M., Notas de asedio al habla de Málaga, Tirada Aparte de Málaga, 16 y 17, Ayuntamiento de Málaga, Málaga, 1973; reed. in Puerta Nueva, Rev. de Educación, Consejería de Educación de la Junta de Andalucía, $n^{\circ}$ 17, marzo, 1993, 6-13.

- AEVAR, M., «Sevilla, macrocosmos lingüístico. Fonética y fonología según el Atlas Lingüístico y Etnográfico de Andalucía», in Homenaje a Ángel Rosenblat en sus 70 años, Instituto Pedagógico, Caracas, 1974, 13-92.

- AvilA, A., La variación reticular e individual en el vernáculo urbano malagueño. Datos del barrio de Capuchinos. Memoria de Licenciatura Inédita/ Departamento de Filología Española I, Universidad de Málaga, 1994

- CARAVEDO, R., « ¿Restos de distinción /s//0/ en el español del Perú?», RFE 72 (1992), 639-654.

- CARBONERO, P., «Norma estándar y actitud sociolingüística», en Sociolingüística andaluza. Metodología y estudios, Universidad de Sevilla, [985 [1982], 141-150.

- CARBONERO, P., «Aspectos sociolingüísticos sobre la nivelación en el español meridional», RFR 3 (1985), 77-83.

- CARBONERO, P. et alli, El habla de Jerez. Estudio sociolingüístico, BUC, Cuadernos de Divulgación, Ayuntamiento de Jerez, 1992.

- GALEOTE, M., El habla mural del ireviño de Iznájar, Villanueva de Tapiay Venta de Santa Bárbara, Ayunt. de Iznájar -Ed. TAT, Granada, 1988.

- LLORENTE MALDONADO, A. «Fonética y fonología andaluzas», RFE 45 (1962), 227-240.

- MOYA J. A., Y E. GARCÍA WIEDEMANN (en prensa), «Seseo, ceceo y distinción en Granada».

- NAVARRO TOMÁS, T., A. M. ESPINOSA, HIJO, Y L. RODRIGUEZ CASTELLANO, «La frontera del andaluz», in Capitulos de Geografía 
Linguística de la Peninsula Ibérica, Publ. del Instituto Caro y Cuervo, Bogotá, 1975, 20-80.

- SALVADOR, F., «Niveles sociolingüísticos de seseo, ceceo y distinción en la ciudad de Granada», EA 37-38 (1980), 25-32.

- SAWOFF, A., «A sociolinguistic appraisal of the sibilant pronunciation in the city of Seville.» Festgabe für Norman Denison, Grazer Linguistische Studien 11-12, 1980, 238-262.

- URUBURU, A., «Seseo en el habla juvenil de la ciudad de Córdoba (España)», in Estudios sobre la lengua española en Córdoba, Diputación Provincial, Córdoba, 1990, 125-134.

- VILlENA, J. A., La ciudad lingüistica. Fundamentos criticos de la sociolingüística urbana. Servicio de Publicaciones, Universidad de Granada, 1994.

- VILLENA, J. A. Y F. REQUENA, «Género, educación y uso lingüístico: la variación social y reticular de $s$ y $z$ en la ciudad de Málaga», en prensá, 1994. 